\title{
Living Isospecific Styrene Polymerization By Chiral Benzyl Titanium Complexes That Contain a Tetradentate [OSSO]-Type Bis(phenolato) Ligand
}

\author{
Klaus Beckerle, Ramanujachary Manivannan, Thomas P. Spaniol, and Jun Okuda*
}

Institut für Anorganische Chemie, RWTH Aachen University, Landoltweg 1,

$$
\text { D-52056 Aachen, Germany }
$$

Table S1. Experimental Data for the Crystal Structure Determinations of $\mathbf{2 b}$

Table S2. Atomic coordinates $\left(\times 10^{4}\right)$ and equivalent isotropic displacement parameters $\left(\AA^{2} \times\right.$ $10^{3}$ ) for $\mathbf{2 b .}$

Table S3. Bond lengths $[\AA]$ and angles $\left[{ }^{\circ}\right]$ for $\mathbf{2 b}$.

Table S6. Torsion angles $\left[{ }^{\circ}\right]$ for $\mathbf{2 b}$.

Table S4. Anisotropic displacement parameters $\left(\AA^{2} \times 10^{3}\right)$ for $\mathbf{2 b}$.

Table S5. Hydrogen coordinates $\left(\times 10^{4}\right)$ and isotropic displacement parameters $\left(\AA^{2} \times 10^{3}\right)$ for $2 \mathbf{b}$.

Table S6. Torsion angles $\left[{ }^{\circ}\right]$ for $\mathbf{2 b}$.

Figure S1. ORTEP diagram of the molecular structure of $\mathbf{2 b}$. Hydrogen atoms were omitted for clarity; thermal ellipsoids are drawn at the $50 \%$ probability level.

Figure S2a. Variable-temperature ${ }^{1} \mathrm{H}$ NMR spectrum of the reaction mixture of $\mathbf{2 a}$ with $\mathrm{B}\left(\mathrm{C}_{6} \mathrm{~F}_{5}\right)_{3}$.

Figure S2b. Variable-temperature ${ }^{13} \mathrm{C}$ NMR spectrum of the reaction mixture of $\mathbf{2 a}$ with $\mathrm{B}\left(\mathrm{C}_{6} \mathrm{~F}_{5}\right)_{3}$.

Figure S3a. ${ }^{1} \mathrm{H}$ NMR spectrum of isotactic polystyrene obtained by titanium complex $\mathbf{2 a}$ activated with $\left[\mathrm{PhNMe}_{2} \mathrm{H}\right]\left[\mathrm{B}\left(\mathrm{C}_{6} \mathrm{~F}_{5}\right)_{4}\right] / \mathrm{Al}^{\mathrm{n}} \mathrm{Oct}_{3}$ (run 6, Table 3).

Figure S3a. ${ }^{13} \mathrm{C}$ NMR spectrum of isotactic polystyrene obtained by titanium complex $2 \mathrm{a}$ activated with $\left[\mathrm{PhNMe}_{2} \mathrm{H}\right]\left[\mathrm{B}\left(\mathrm{C}_{6} \mathrm{~F}_{5}\right)_{4}\right] / \mathrm{Al}^{\mathrm{n}} \mathrm{Oct}_{3}$ (run 6, Table 3). 
Table S1. Experimental Data for the Crystal Structure Determinations of 2b

\begin{tabular}{ll}
\hline \multicolumn{1}{c}{ Crystal data and Data collection } & \\
formula & $\mathrm{C}_{47.50} \mathrm{H}_{62} \mathrm{O}_{2} \mathrm{~S}_{2} \mathrm{Ti}$ \\
fw & 777.02 \\
crystal size & $0.63 \times 0.26 \times 0.14 \mathrm{~mm}$ \\
crystal color & dark red \\
crystal system & triclinic \\
space group & $\mathrm{P}-1$ (no. 2$)$ \\
$a, \AA$ & $9.4075(18)$ \\
$b, \AA$ & $15.683(3)$ \\
$c, \AA$ & $16.292(3)$ \\
$\alpha$, deg & $104.389(5)$ \\
$\beta$, deg & $102.912(5)$ \\
$\gamma$, deg & $103.148(5)$ \\
$V, \AA^{3}$ & $2165.1(7)$ \\
$Z$ & 2 \\
$\rho_{\text {calcd }}$ gcm & \\
$\mu$, mm & -1
\end{tabular}


Table S2. Atomic coordinates $\left(\times 10^{4}\right)$ and equivalent isotropic displacement parameters $\left(\AA^{2} \times\right.$ $10^{3}$ ) for $\mathbf{2 b}$. U(eq) is defined as one third of the trace of the orthogonalized Uij tensor.

\begin{tabular}{|c|c|c|c|c|}
\hline & $\mathrm{X}$ & $\mathrm{y}$ & $\mathrm{Z}$ & $\mathrm{U}(\mathrm{eq})$ \\
\hline $\mathrm{Ti}$ & $2037(1)$ & $2557(1)$ & 2441(1) & $17(1)$ \\
\hline $\mathrm{S}(1)$ & $4466(1)$ & $3092(1)$ & 1689(1) & $22(1)$ \\
\hline$S(2)$ & $4710(1)$ & $3034(1)$ & $3785(1)$ & $20(1)$ \\
\hline $\mathrm{O}(1)$ & $2446(1)$ & $3823(1)$ & 2599(1) & $19(1)$ \\
\hline $\mathrm{O}(2)$ & $2458(1)$ & $1451(1)$ & $2472(1)$ & $19(1)$ \\
\hline $\mathrm{C}(1)$ & $3347(2)$ & $4484(1)$ & 2384(1) & $17(1)$ \\
\hline $\mathrm{C}(2)$ & $4435(2)$ & $4263(1)$ & 1964(1) & $19(1)$ \\
\hline $\mathrm{C}(3)$ & $5409(2)$ & $4923(1)$ & $1736(1)$ & 21(1) \\
\hline $\mathrm{C}(4)$ & $5322(2)$ & $5825(1)$ & $1917(1)$ & $22(1)$ \\
\hline $\mathrm{C}(5)$ & $4202(2)$ & $6025(1)$ & 2311(1) & 21(1) \\
\hline$C(6)$ & $3207(2)$ & $5390(1)$ & 2552(1) & $18(1)$ \\
\hline $\mathrm{C}(7)$ & $6385(2)$ & $6560(2)$ & $1664(2)$ & 29(1) \\
\hline $\mathrm{C}(8 \mathrm{~A})$ & $6008(4)$ & $6242(2)$ & $629(2)$ & $38(1)$ \\
\hline $\mathrm{C}(9 \mathrm{~A})$ & $8041(4)$ & $6656(3)$ & 2071(3) & $41(1)$ \\
\hline $\mathrm{C}(10 \mathrm{~A})$ & $6097(4)$ & $7504(2)$ & $1929(2)$ & $40(1)$ \\
\hline $\mathrm{C}(8 \mathrm{~B})$ & $5625(16)$ & $6848(10)$ & $1017(11)$ & $55(4)$ \\
\hline $\mathrm{C}(9 \mathrm{~B})$ & $7766(18)$ & $6231(11)$ & 1411(14) & $58(4)$ \\
\hline $\mathrm{C}(10 \mathrm{~B})$ & $7290(20)$ & $7438(10)$ & $2570(10)$ & ) $75(6)$ \\
\hline $\mathrm{C}(11)$ & $2035(2)$ & $5661(1)$ & 3014(1) & $21(1)$ \\
\hline $\mathrm{C}(12)$ & $405(2)$ & $5002(1)$ & $2512(1)$ & $24(1)$ \\
\hline $\mathrm{C}(13)$ & $2532(3)$ & $5635(2)$ & $3975(1)$ & $29(1)$ \\
\hline $\mathrm{C}(14)$ & 1984(3) & $6646(1)$ & $3057(2)$ & $32(1)$ \\
\hline $\mathrm{C}(15)$ & $6236(2)$ & $3180(1)$ & $2495(2)$ & $27(1)$ \\
\hline$C(16)$ & $6292(2)$ & $3603(1)$ & $3448(2)$ & $27(1)$ \\
\hline $\mathrm{C}(17)$ & $4836(2)$ & 1884(1) & $3577(1)$ & 19(1) \\
\hline $\mathrm{C}(18)$ & $3599(2)$ & 1194(1) & $2915(1)$ & $18(1)$ \\
\hline C(19) & $3558(2)$ & $258(1)$ & 2744(1) & $18(1)$ \\
\hline$C(20)$ & $4816(2)$ & $81(1)$ & $3237(1)$ & $20(1)$ \\
\hline $\mathrm{C}(21)$ & $6071(2)$ & 761(1) & $3902(1)$ & 19(1) \\
\hline$C(22)$ & $6049(2)$ & $1673(1)$ & 4074(1) & $20(1)$ \\
\hline $\mathrm{C}(23)$ & $2153(2)$ & $-522(1)$ & $2075(1)$ & $22(1)$ \\
\hline $\mathrm{C}(24)$ & $754(2)$ & $-499(2)$ & $2413(2)$ & $31(1)$ \\
\hline$C(25)$ & $1828(3)$ & $-412(1)$ & $1142(1)$ & $30(1)$ \\
\hline$C(26)$ & $2372(3)$ & $-1479(1)$ & 1987(2) & $35(1)$ \\
\hline $\mathrm{C}(27)$ & $7410(2)$ & 482(1) & $4394(1)$ & $22(1)$ \\
\hline $\mathrm{C}(28)$ & $8273(2)$ & $164(2)$ & $3732(1)$ & $28(1)$ \\
\hline C(29) & $6813(3)$ & $-322(2)$ & $4737(2)$ & $32(1)$ \\
\hline$C(30)$ & $8521(2)$ & $1290(2)$ & $5190(1)$ & $30(1)$ \\
\hline $\mathrm{C}(31)$ & $728(2)$ & 1996(1) & $1064(1)$ & $22(1)$ \\
\hline $\mathrm{C}(32)$ & $-92(2)$ & $2588(1)$ & $696(1)$ & 21(1) \\
\hline$C(33)$ & $616(2)$ & $3241(1)$ & $342(1)$ & $25(1)$ \\
\hline $\mathrm{C}(34)$ & $-162(2)$ & $3782(1)$ & $-16(1)$ & $28(1)$ \\
\hline$C(35)$ & $-1671(3)$ & $3698(2)$ & $-23(1)$ & $30(1)$ \\
\hline $\mathrm{C}(36)$ & $-2386(2)$ & $3066(2)$ & $334(1)$ & $29(1)$ \\
\hline $\mathrm{C}(37)$ & $-1614(2)$ & $2520(1)$ & $686(1)$ & $25(1)$ \\
\hline $\mathrm{C}(38)$ & $-11(2)$ & $2283(1)$ & 2821(1) & $23(1)$ \\
\hline C(39) & $707(2)$ & 2686(1) & 3793(1) & $24(1)$ \\
\hline
\end{tabular}




\begin{tabular}{lllll}
\hline $\mathrm{C}(40)$ & $1589(2)$ & $2259(2)$ & $4286(2)$ & $29(1)$ \\
$\mathrm{C}(41)$ & $2370(3)$ & $2685(2)$ & $5183(2)$ & $37(1)$ \\
$\mathrm{C}(42)$ & $2272(3)$ & $3546(2)$ & $5625(2)$ & $42(1)$ \\
$\mathrm{C}(43)$ & $1384(3)$ & $3967(2)$ & $5159(2)$ & $38(1)$ \\
$\mathrm{C}(44)$ & $630(2)$ & $3551(1)$ & $4260(2)$ & $29(1)$ \\
$\mathrm{C}(45)$ & $5380(6)$ & $-102(5)$ & $210(5)$ & $51(2)$ \\
$\mathrm{C}(46)$ & $4830(20)$ & $635(15)$ & $384(9)$ & $60(4)$ \\
$\mathrm{C}(47)$ & $3848(9)$ & $846(4)$ & $-282(5)$ & $57(2)$ \\
$\mathrm{C}(48)$ & $3481(18)$ & $281(10)$ & $-1124(11)$ & $46(3)$ \\
$\mathrm{C}(49)$ & $3950(6)$ & $-499(4)$ & $-1325(4)$ & $39(1)$ \\
$\mathrm{C}(50)$ & $4904(19)$ & $-695(12)$ & $-657(9)$ & $43(3)$ \\
$\mathrm{C}(51)$ & $6510(20)$ & $-239(15)$ & $961(14)$ & $100(7)$ \\
\hline
\end{tabular}


Table S3. Bond lengths $[\AA]$ and angles $\left[{ }^{\circ}\right]$ for $\mathbf{2 b}$.

\begin{tabular}{|c|c|}
\hline $\mathrm{Ti}-\mathrm{O}(1)$ & $1.8732(13)$ \\
\hline $\mathrm{Ti}-\mathrm{O}(2)$ & $1.8758(13)$ \\
\hline Ti-C(38) & $2.137(2)$ \\
\hline Ti-C(31) & $2.149(2)$ \\
\hline $\mathrm{Ti}-\mathrm{S}(2)$ & $2.7472(7)$ \\
\hline $\mathrm{Ti}-\mathrm{S}(1)$ & $2.8836(7)$ \\
\hline$S(1)-C(2)$ & $1.7874(19)$ \\
\hline$S(1)-C(15)$ & $1.829(2)$ \\
\hline$S(2)-C(17)$ & $1.7869(19)$ \\
\hline$S(2)-C(16)$ & $1.820(2)$ \\
\hline $\mathrm{O}(1)-\mathrm{C}(1)$ & $1.353(2)$ \\
\hline $\mathrm{O}(2)-\mathrm{C}(18)$ & $1.353(2)$ \\
\hline$C(1)-C(2)$ & $1.412(3)$ \\
\hline$C(1)-C(6)$ & $1.421(3)$ \\
\hline$C(2)-C(3)$ & $1.401(3)$ \\
\hline$C(3)-C(4)$ & $1.396(3)$ \\
\hline $\mathrm{C}(3)-\mathrm{H}(3)$ & 0.9500 \\
\hline$C(4)-C(5)$ & $1.404(3)$ \\
\hline$C(4)-C(7)$ & $1.549(3)$ \\
\hline$C(5)-C(6)$ & $1.399(3)$ \\
\hline $\mathrm{C}(5)-\mathrm{H}(5)$ & 0.9500 \\
\hline$C(6)-C(11)$ & $1.545(3)$ \\
\hline $\mathrm{C}(7)-\mathrm{C}(8 \mathrm{~B})$ & $1.369(14)$ \\
\hline $\mathrm{C}(7)-\mathrm{C}(9 \mathrm{~A})$ & $1.507(4)$ \\
\hline$C(7)-C(10 A)$ & $1.539(3)$ \\
\hline $\mathrm{C}(7)-\mathrm{C}(8 \mathrm{~A})$ & $1.565(4)$ \\
\hline $\mathrm{C}(7)-\mathrm{C}(9 \mathrm{~B})$ & $1.600(15)$ \\
\hline $\mathrm{C}(7)-\mathrm{C}(10 \mathrm{~B})$ & $1.641(13)$ \\
\hline $\mathrm{C}(8 \mathrm{~A})-\mathrm{H}(8 \mathrm{~A})$ & 0.9800 \\
\hline $\mathrm{C}(8 \mathrm{~A})-\mathrm{H}(8 \mathrm{~B})$ & 0.9800 \\
\hline $\mathrm{C}(8 \mathrm{~A})-\mathrm{H}(8 \mathrm{C})$ & 0.9800 \\
\hline $\mathrm{C}(9 \mathrm{~A})-\mathrm{H}(9 \mathrm{~A})$ & 0.9800 \\
\hline $\mathrm{C}(9 \mathrm{~A})-\mathrm{H}(9 \mathrm{~B})$ & 0.9800 \\
\hline $\mathrm{C}(9 \mathrm{~A})-\mathrm{H}(9 \mathrm{C})$ & 0.9800 \\
\hline $\mathrm{C}(10 \mathrm{~A})-\mathrm{H}(10 \mathrm{~A})$ & 0.9800 \\
\hline $\mathrm{C}(10 \mathrm{~A})-\mathrm{H}(10 \mathrm{~B})$ & 0.9800 \\
\hline $\mathrm{C}(10 \mathrm{~A})-\mathrm{H}(10 \mathrm{C})$ & 0.9800 \\
\hline $\mathrm{C}(8 \mathrm{~B})-\mathrm{H}(8 \mathrm{D})$ & 0.9800 \\
\hline $\mathrm{C}(8 \mathrm{~B})-\mathrm{H}(8 \mathrm{E})$ & 0.9800 \\
\hline $\mathrm{C}(8 \mathrm{~B})-\mathrm{H}(8 \mathrm{~F})$ & 0.9800 \\
\hline $\mathrm{C}(9 \mathrm{~B})-\mathrm{H}(9 \mathrm{D})$ & 0.9800 \\
\hline $\mathrm{C}(9 \mathrm{~B})-\mathrm{H}(9 \mathrm{E})$ & 0.9800 \\
\hline $\mathrm{C}(9 \mathrm{~B})-\mathrm{H}(9 \mathrm{~F})$ & 0.9800 \\
\hline $\mathrm{C}(10 \mathrm{~B})-\mathrm{H}(10 \mathrm{D})$ & 0.9800 \\
\hline $\mathrm{C}(10 \mathrm{~B})-\mathrm{H}(10 \mathrm{E})$ & 0.9800 \\
\hline $\mathrm{C}(10 \mathrm{~B})-\mathrm{H}(10 \mathrm{~F})$ & 0.9800 \\
\hline $\mathrm{C}(11)-\mathrm{C}(14)$ & $1.541(3)$ \\
\hline $\mathrm{C}(11)-\mathrm{C}(13)$ & $1.545(3)$ \\
\hline $\mathrm{C}(11)-\mathrm{C}(12)$ & $1.545(3)$ \\
\hline
\end{tabular}




\begin{tabular}{|c|c|}
\hline $\mathrm{C}(12)-\mathrm{H}(12 \mathrm{~A})$ & 0.9800 \\
\hline $\mathrm{C}(12)-\mathrm{H}(12 \mathrm{~B})$ & 0.9800 \\
\hline $\mathrm{C}(12)-\mathrm{H}(12 \mathrm{C})$ & 0.9800 \\
\hline $\mathrm{C}(13)-\mathrm{H}(13 \mathrm{~A})$ & 0.9800 \\
\hline $\mathrm{C}(13)-\mathrm{H}(13 \mathrm{~B})$ & 0.9800 \\
\hline $\mathrm{C}(13)-\mathrm{H}(13 \mathrm{C})$ & 0.9800 \\
\hline $\mathrm{C}(14)-\mathrm{H}(14 \mathrm{~A})$ & 0.9800 \\
\hline $\mathrm{C}(14)-\mathrm{H}(14 \mathrm{~B})$ & 0.9800 \\
\hline $\mathrm{C}(14)-\mathrm{H}(14 \mathrm{C})$ & 0.9800 \\
\hline$C(15)-C(16)$ & $1.514(3)$ \\
\hline $\mathrm{C}(15)-\mathrm{H}(15 \mathrm{~A})$ & 0.9900 \\
\hline $\mathrm{C}(15)-\mathrm{H}(15 \mathrm{~B})$ & 0.9900 \\
\hline $\mathrm{C}(16)-\mathrm{H}(16 \mathrm{~A})$ & 0.9900 \\
\hline $\mathrm{C}(16)-\mathrm{H}(16 \mathrm{~B})$ & 0.9900 \\
\hline $\mathrm{C}(17)-\mathrm{C}(22)$ & $1.400(3)$ \\
\hline $\mathrm{C}(17)-\mathrm{C}(18)$ & $1.405(3)$ \\
\hline $\mathrm{C}(18)-\mathrm{C}(19)$ & $1.415(3)$ \\
\hline $\mathrm{C}(19)-\mathrm{C}(20)$ & $1.399(3)$ \\
\hline $\mathrm{C}(19)-\mathrm{C}(23)$ & $1.545(3)$ \\
\hline $\mathrm{C}(20)-\mathrm{C}(21)$ & $1.409(3)$ \\
\hline $\mathrm{C}(20)-\mathrm{H}(20)$ & 0.9500 \\
\hline $\mathrm{C}(21)-\mathrm{C}(22)$ & $1.393(3)$ \\
\hline $\mathrm{C}(21)-\mathrm{C}(27)$ & $1.547(3)$ \\
\hline $\mathrm{C}(22)-\mathrm{H}(22)$ & 0.9500 \\
\hline $\mathrm{C}(23)-\mathrm{C}(26)$ & $1.537(3)$ \\
\hline $\mathrm{C}(23)-\mathrm{C}(24)$ & $1.540(3)$ \\
\hline $\mathrm{C}(23)-\mathrm{C}(25)$ & $1.544(3)$ \\
\hline $\mathrm{C}(24)-\mathrm{H}(24 \mathrm{~A})$ & 0.9800 \\
\hline $\mathrm{C}(24)-\mathrm{H}(24 \mathrm{~B})$ & 0.9800 \\
\hline $\mathrm{C}(24)-\mathrm{H}(24 \mathrm{C})$ & 0.9800 \\
\hline $\mathrm{C}(25)-\mathrm{H}(25 \mathrm{~A})$ & 0.9800 \\
\hline $\mathrm{C}(25)-\mathrm{H}(25 \mathrm{~B})$ & 0.9800 \\
\hline $\mathrm{C}(25)-\mathrm{H}(25 \mathrm{C})$ & 0.9800 \\
\hline $\mathrm{C}(26)-\mathrm{H}(26 \mathrm{~A})$ & 0.9800 \\
\hline $\mathrm{C}(26)-\mathrm{H}(26 \mathrm{~B})$ & 0.9800 \\
\hline $\mathrm{C}(26)-\mathrm{H}(26 \mathrm{C})$ & 0.9800 \\
\hline$C(27)-C(30)$ & $1.534(3)$ \\
\hline $\mathrm{C}(27)-\mathrm{C}(28)$ & $1.542(3)$ \\
\hline $\mathrm{C}(27)-\mathrm{C}(29)$ & $1.545(3)$ \\
\hline $\mathrm{C}(28)-\mathrm{H}(28 \mathrm{~A})$ & 0.9800 \\
\hline $\mathrm{C}(28)-\mathrm{H}(28 \mathrm{~B})$ & 0.9800 \\
\hline $\mathrm{C}(28)-\mathrm{H}(28 \mathrm{C})$ & 0.9800 \\
\hline $\mathrm{C}(29)-\mathrm{H}(29 \mathrm{~A})$ & 0.9800 \\
\hline $\mathrm{C}(29)-\mathrm{H}(29 \mathrm{~B})$ & 0.9800 \\
\hline $\mathrm{C}(29)-\mathrm{H}(29 \mathrm{C})$ & 0.9800 \\
\hline $\mathrm{C}(30)-\mathrm{H}(30 \mathrm{~A})$ & 0.9800 \\
\hline $\mathrm{C}(30)-\mathrm{H}(30 \mathrm{~B})$ & 0.9800 \\
\hline $\mathrm{C}(30)-\mathrm{H}(30 \mathrm{C})$ & 0.9800 \\
\hline $\mathrm{C}(31)-\mathrm{C}(32)$ & $1.487(3)$ \\
\hline $\mathrm{C}(31)-\mathrm{C}(38)$ & $3.046(3)$ \\
\hline $\mathrm{C}(31)-\mathrm{H}(31 \mathrm{~A})$ & 0.9900 \\
\hline
\end{tabular}




\begin{tabular}{|c|c|}
\hline $\mathrm{C}(31)-\mathrm{H}(31 \mathrm{~B})$ & 0.9900 \\
\hline $\mathrm{C}(32)-\mathrm{C}(33)$ & $1.407(3)$ \\
\hline $\mathrm{C}(32)-\mathrm{C}(37)$ & $1.408(3)$ \\
\hline $\mathrm{C}(33)-\mathrm{C}(34)$ & $1.390(3)$ \\
\hline $\mathrm{C}(33)-\mathrm{H}(33)$ & 0.9500 \\
\hline $\mathrm{C}(34)-\mathrm{C}(35)$ & $1.393(3)$ \\
\hline $\mathrm{C}(34)-\mathrm{H}(34)$ & 0.9500 \\
\hline $\mathrm{C}(35)-\mathrm{C}(36)$ & $1.390(3)$ \\
\hline $\mathrm{C}(35)-\mathrm{H}(35)$ & 0.9500 \\
\hline $\mathrm{C}(36)-\mathrm{C}(37)$ & $1.388(3)$ \\
\hline $\mathrm{C}(36)-\mathrm{H}(36)$ & 0.9500 \\
\hline $\mathrm{C}(37)-\mathrm{H}(37)$ & 0.9500 \\
\hline $\mathrm{C}(38)-\mathrm{C}(39)$ & $1.480(3)$ \\
\hline $\mathrm{C}(38)-\mathrm{H}(38 \mathrm{~A})$ & 0.9900 \\
\hline $\mathrm{C}(38)-\mathrm{H}(38 \mathrm{~B})$ & 0.9900 \\
\hline$C(39)-C(44)$ & $1.408(3)$ \\
\hline$C(39)-C(40)$ & $1.416(3)$ \\
\hline $\mathrm{C}(40)-\mathrm{C}(41)$ & $1.391(3)$ \\
\hline $\mathrm{C}(40)-\mathrm{H}(40)$ & 0.9500 \\
\hline $\mathrm{C}(41)-\mathrm{C}(42)$ & $1.397(4)$ \\
\hline $\mathrm{C}(41)-\mathrm{H}(41)$ & 0.9500 \\
\hline $\mathrm{C}(42)-\mathrm{C}(43)$ & $1.389(3)$ \\
\hline $\mathrm{C}(42)-\mathrm{H}(42)$ & 0.9500 \\
\hline $\mathrm{C}(43)-\mathrm{C}(44)$ & $1.387(3)$ \\
\hline $\mathrm{C}(43)-\mathrm{H}(43)$ & 0.9500 \\
\hline $\mathrm{C}(44)-\mathrm{H}(44)$ & 0.9500 \\
\hline$C(45)-C(45) \# 1$ & $1.037(11)$ \\
\hline $\mathrm{C}(45)-\mathrm{C}(46) \# 1$ & $1.05(2)$ \\
\hline$C(45)-C(46)$ & $1.362(19)$ \\
\hline $\mathrm{C}(45)-\mathrm{C}(50)$ & $1.392(13)$ \\
\hline$C(45)-C(50) \# 1$ & $1.40(2)$ \\
\hline $\mathrm{C}(45)-\mathrm{C}(47) \# 1$ & $1.522(10)$ \\
\hline $\mathrm{C}(45)-\mathrm{C}(51)$ & $1.526(18)$ \\
\hline $\mathrm{C}(46)-\mathrm{C}(47)$ & $1.418(19)$ \\
\hline $\mathrm{C}(46)-\mathrm{H}(46)$ & 0.9500 \\
\hline $\mathrm{C}(47)-\mathrm{C}(48)$ & $1.353(18)$ \\
\hline $\mathrm{C}(47)-\mathrm{H}(47)$ & 0.9500 \\
\hline $\mathrm{C}(48)-\mathrm{C}(49)$ & $1.381(13)$ \\
\hline $\mathrm{C}(48)-\mathrm{H}(48)$ & 0.9500 \\
\hline$C(49)-C(50)$ & $1.393(16)$ \\
\hline $\mathrm{C}(49)-\mathrm{H}(49)$ & 0.9500 \\
\hline $\mathrm{C}(50)-\mathrm{H}(50)$ & 0.9500 \\
\hline $\mathrm{C}(51)-\mathrm{H}(51 \mathrm{~A})$ & 0.9800 \\
\hline $\mathrm{C}(51)-\mathrm{H}(51 \mathrm{~B})$ & 0.9800 \\
\hline $\mathrm{C}(51)-\mathrm{H}(51 \mathrm{C})$ & 0.9800 \\
\hline $\mathrm{O}(1)-\mathrm{Ti}-\mathrm{O}(2)$ & $157.55(6)$ \\
\hline $\mathrm{O}(1)-\mathrm{Ti}-\mathrm{C}(38)$ & $100.77(7)$ \\
\hline $\mathrm{O}(2)-\mathrm{Ti}-\mathrm{C}(38)$ & $96.71(7)$ \\
\hline $\mathrm{O}(1)-\mathrm{Ti}-\mathrm{C}(31)$ & $100.59(7)$ \\
\hline $\mathrm{O}(2)-\mathrm{Ti}-\mathrm{C}(31)$ & $93.18(7)$ \\
\hline
\end{tabular}




\begin{tabular}{|c|c|}
\hline $\mathrm{C}(38)-\mathrm{Ti}-\mathrm{C}(31)$ & $90.57(8)$ \\
\hline $\mathrm{O}(1)-\mathrm{Ti}-\mathrm{S}(2)$ & $86.06(4)$ \\
\hline $\mathrm{O}(2)-\mathrm{Ti}-\mathrm{S}(2)$ & $73.69(4)$ \\
\hline $\mathrm{C}(38)-\mathrm{Ti}-\mathrm{S}(2)$ & $115.45(6)$ \\
\hline C(31)-Ti-S(2) & $151.66(6)$ \\
\hline $\mathrm{O}(1)-\mathrm{Ti}-\mathrm{S}(1)$ & $72.23(4)$ \\
\hline $\mathrm{O}(2)-\mathrm{Ti}-\mathrm{S}(1)$ & $92.57(4)$ \\
\hline $\mathrm{C}(38)-\mathrm{Ti}-\mathrm{S}(1)$ & $168.23(6)$ \\
\hline C(31)-Ti-S(1) & $81.67(6)$ \\
\hline $\mathrm{S}(2)-\mathrm{Ti}-\mathrm{S}(1)$ & $74.14(2)$ \\
\hline$C(2)-S(1)-C(15)$ & $101.88(9)$ \\
\hline $\mathrm{C}(2)-\mathrm{S}(1)-\mathrm{Ti}$ & $92.27(7)$ \\
\hline $\mathrm{C}(15)-\mathrm{S}(1)-\mathrm{Ti}$ & $106.27(7)$ \\
\hline$C(17)-S(2)-C(16)$ & $102.50(9)$ \\
\hline $\mathrm{C}(17)-\mathrm{S}(2)-\mathrm{Ti}$ & $94.62(6)$ \\
\hline $\mathrm{C}(16)-\mathrm{S}(2)-\mathrm{Ti}$ & $109.18(7)$ \\
\hline $\mathrm{C}(1)-\mathrm{O}(1)-\mathrm{Ti}$ & $138.90(12)$ \\
\hline $\mathrm{C}(18)-\mathrm{O}(2)-\mathrm{Ti}$ & 137.14(12) \\
\hline $\mathrm{O}(1)-\mathrm{C}(1)-\mathrm{C}(2)$ & $119.07(16)$ \\
\hline $\mathrm{O}(1)-\mathrm{C}(1)-\mathrm{C}(6)$ & $121.84(16)$ \\
\hline$C(2)-C(1)-C(6)$ & $119.08(17)$ \\
\hline$C(3)-C(2)-C(1)$ & $121.46(17)$ \\
\hline$C(3)-C(2)-S(1)$ & $121.11(15)$ \\
\hline$C(1)-C(2)-S(1)$ & $117.37(14)$ \\
\hline $\mathrm{C}(4)-\mathrm{C}(3)-\mathrm{C}(2)$ & $120.48(18)$ \\
\hline $\mathrm{C}(4)-\mathrm{C}(3)-\mathrm{H}(3)$ & 119.8 \\
\hline $\mathrm{C}(2)-\mathrm{C}(3)-\mathrm{H}(3)$ & 119.8 \\
\hline$C(3)-C(4)-C(5)$ & $117.24(18)$ \\
\hline$C(3)-C(4)-C(7)$ & $121.00(18)$ \\
\hline$C(5)-C(4)-C(7)$ & $121.73(18)$ \\
\hline$C(6)-C(5)-C(4)$ & $124.35(18)$ \\
\hline $\mathrm{C}(6)-\mathrm{C}(5)-\mathrm{H}(5)$ & 117.8 \\
\hline $\mathrm{C}(4)-\mathrm{C}(5)-\mathrm{H}(5)$ & 117.8 \\
\hline $\mathrm{C}(5)-\mathrm{C}(6)-\mathrm{C}(1)$ & $117.34(17)$ \\
\hline$C(5)-C(6)-C(11)$ & $121.95(17)$ \\
\hline$C(1)-C(6)-C(11)$ & $120.68(16)$ \\
\hline $\mathrm{C}(8 \mathrm{~B})-\mathrm{C}(7)-\mathrm{C}(9 \mathrm{~A})$ & $134.4(6)$ \\
\hline $\mathrm{C}(8 \mathrm{~B})-\mathrm{C}(7)-\mathrm{C}(10 \mathrm{~A})$ & $61.0(7)$ \\
\hline$C(9 A)-C(7)-C(10 A)$ & $110.2(2)$ \\
\hline $\mathrm{C}(8 \mathrm{~B})-\mathrm{C}(7)-\mathrm{C}(4)$ & $113.5(6)$ \\
\hline $\mathrm{C}(9 \mathrm{~A})-\mathrm{C}(7)-\mathrm{C}(4)$ & $110.98(19)$ \\
\hline$C(10 A)-C(7)-C(4)$ & $112.12(19)$ \\
\hline $\mathrm{C}(8 \mathrm{~B})-\mathrm{C}(7)-\mathrm{C}(8 \mathrm{~A})$ & $46.8(7)$ \\
\hline $\mathrm{C}(9 \mathrm{~A})-\mathrm{C}(7)-\mathrm{C}(8 \mathrm{~A})$ & 109.1(2) \\
\hline$C(10 A)-C(7)-C(8 A)$ & $106.3(2)$ \\
\hline $\mathrm{C}(4)-\mathrm{C}(7)-\mathrm{C}(8 \mathrm{~A})$ & 107.97(19) \\
\hline $\mathrm{C}(8 \mathrm{~B})-\mathrm{C}(7)-\mathrm{C}(9 \mathrm{~B})$ & $111.3(9)$ \\
\hline $\mathrm{C}(9 \mathrm{~A})-\mathrm{C}(7)-\mathrm{C}(9 \mathrm{~B})$ & $39.4(7)$ \\
\hline $\mathrm{C}(10 \mathrm{~A})-\mathrm{C}(7)-\mathrm{C}(9 \mathrm{~B})$ & 133.9(6) \\
\hline $\mathrm{C}(4)-\mathrm{C}(7)-\mathrm{C}(9 \mathrm{~B})$ & $112.0(5)$ \\
\hline $\mathrm{C}(8 \mathrm{~A})-\mathrm{C}(7)-\mathrm{C}(9 \mathrm{~B})$ & $71.7(7)$ \\
\hline
\end{tabular}




\begin{tabular}{lc} 
& \\
\hline $\mathrm{C}(8 \mathrm{~B})-\mathrm{C}(7)-\mathrm{C}(10 \mathrm{~B})$ & $110.2(10)$ \\
$\mathrm{C}(9 \mathrm{~A})-\mathrm{C}(7)-\mathrm{C}(10 \mathrm{~B})$ & $64.4(8)$ \\
$\mathrm{C}(10 \mathrm{~A})-\mathrm{C}(7)-\mathrm{C}(10 \mathrm{~B})$ & $51.8(8)$ \\
$\mathrm{C}(4)-\mathrm{C}(7)-\mathrm{C}(10 \mathrm{~B})$ & $107.3(5)$ \\
$\mathrm{C}(8 \mathrm{~A})-\mathrm{C}(7)-\mathrm{C}(10 \mathrm{~B})$ & $143.8(5)$ \\
$\mathrm{C}(9 \mathrm{~B})-\mathrm{C}(7)-\mathrm{C}(10 \mathrm{~B})$ & $101.9(10)$ \\
$\mathrm{C}(7)-\mathrm{C}(8 \mathrm{~A})-\mathrm{H}(8 \mathrm{~A})$ & 109.5 \\
$\mathrm{C}(7)-\mathrm{C}(8 \mathrm{~A})-\mathrm{H}(8 \mathrm{~B})$ & 109.5 \\
$\mathrm{C}(7)-\mathrm{C}(8 \mathrm{~A})-\mathrm{H}(8 \mathrm{C})$ & 109.5 \\
$\mathrm{C}(7)-\mathrm{C}(9 \mathrm{~A})-\mathrm{H}(9 \mathrm{~A})$ & 109.5 \\
$\mathrm{C}(7)-\mathrm{C}(9 \mathrm{~A})-\mathrm{H}(9 \mathrm{~B})$ & 109.5 \\
$\mathrm{C}(7)-\mathrm{C}(9 \mathrm{~A})-\mathrm{H}(9 \mathrm{C})$ & 109.5 \\
$\mathrm{C}(7)-\mathrm{C}(10 \mathrm{~A})-\mathrm{H}(10 \mathrm{~A})$ & 109.5 \\
$\mathrm{C}(7)-\mathrm{C}(10 \mathrm{~A})-\mathrm{H}(10 \mathrm{~B})$ & 109.5 \\
$\mathrm{C}(7)-\mathrm{C}(10 \mathrm{~A})-\mathrm{H}(10 \mathrm{C})$ & 109.5 \\
$\mathrm{C}(7)-\mathrm{C}(8 \mathrm{~B})-\mathrm{H}(8 \mathrm{D})$ & 109.5 \\
$\mathrm{C}(7)-\mathrm{C}(8 \mathrm{~B})-\mathrm{H}(8 \mathrm{E})$ & 109.5 \\
$\mathrm{H}(8 \mathrm{D})-\mathrm{C}(8 \mathrm{~B})-\mathrm{H}(8 \mathrm{E})$ & 109.5 \\
$\mathrm{C}(7)-\mathrm{C}(8 \mathrm{~B})-\mathrm{H}(8 \mathrm{~F})$ & 109.5 \\
$\mathrm{H}(8 \mathrm{D})-\mathrm{C}(8 \mathrm{~B})-\mathrm{H}(8 \mathrm{~F})$ & 109.5 \\
$\mathrm{H}(8 \mathrm{E})-\mathrm{C}(8 \mathrm{~B})-\mathrm{H}(8 \mathrm{~F})$ & 109.5 \\
$\mathrm{C}(7)-\mathrm{C}(9 \mathrm{~B})-\mathrm{H}(9 \mathrm{D})$ & 109.5 \\
$\mathrm{C}(7)-\mathrm{C}(9 \mathrm{~B})-\mathrm{H}(9 \mathrm{E})$ & 109.5 \\
$\mathrm{H}(9 \mathrm{D})-\mathrm{C}(9 \mathrm{~B})-\mathrm{H}(9 \mathrm{E})$ & 109.5 \\
$\mathrm{C}(7)-\mathrm{C}(9 \mathrm{~B})-\mathrm{H}(9 \mathrm{~F})$ & 109.5 \\
$\mathrm{H}(9 \mathrm{D})-\mathrm{C}(9 \mathrm{~B})-\mathrm{H}(9 \mathrm{~F})$ & 109.5 \\
$\mathrm{H}(9 \mathrm{E})-\mathrm{C}(9 \mathrm{~B})-\mathrm{H}(9 \mathrm{~F})$ & 109.5 \\
$\mathrm{C}(7)-\mathrm{C}(10 \mathrm{~B})-\mathrm{H}(10 \mathrm{D})$ & 109.5 \\
$\mathrm{C}(7)-\mathrm{C}(10 \mathrm{~B})-\mathrm{H}(10 \mathrm{E})$ & 109.5 \\
$\mathrm{H}(10 \mathrm{D})-\mathrm{C}(10 \mathrm{~B})-\mathrm{H}(10 \mathrm{E})$ & 109.5 \\
$\mathrm{C}(7)-\mathrm{C}(10 \mathrm{~B})-\mathrm{H}(10 \mathrm{~F})$ & 109.5 \\
$\mathrm{H}(10 \mathrm{D})-\mathrm{C}(10 \mathrm{~B})-\mathrm{H}(10 \mathrm{~F})$ & 109.5 \\
$\mathrm{H}(10 \mathrm{E})-\mathrm{C}(10 \mathrm{~B})-\mathrm{H}(10 \mathrm{~F})$ & 109.5 \\
$\mathrm{C}(14)-\mathrm{C}(11)-\mathrm{C}(13)$ & $107.94(17)$ \\
$\mathrm{C}(14)-\mathrm{C}(11)-\mathrm{C}(12)$ & $107.26(16)$ \\
$\mathrm{C}(13)-\mathrm{C}(11)-\mathrm{C}(12)$ & $110.69(16)$ \\
$\mathrm{C}(14)-\mathrm{C}(11)-\mathrm{C}(6)$ & $111.76(16)$ \\
$\mathrm{C}(13)-\mathrm{C}(11)-\mathrm{C}(6)$ & $108.04(16)$ \\
$\mathrm{C}(12)-\mathrm{C}(11)-\mathrm{C}(6)$ & $111.12(16)$ \\
$\mathrm{C}(11)-\mathrm{C}(12)-\mathrm{H}(12 \mathrm{~A})$ & 109.5 \\
$\mathrm{C}(11)-\mathrm{C}(12)-\mathrm{H}(12 \mathrm{~B})$ & 109.5 \\
$\mathrm{H}(12 \mathrm{~A})-\mathrm{C}(12)-\mathrm{H}(12 \mathrm{~B})$ & 109.5 \\
$\mathrm{C}(11)-\mathrm{C}(12)-\mathrm{H}(12 \mathrm{C})$ & 109.5 \\
$\mathrm{H}(12 \mathrm{~A})-\mathrm{C}(12)-\mathrm{H}(12 \mathrm{C})$ & 109.5 \\
$\mathrm{H}(12 \mathrm{~B})-\mathrm{C}(12)-\mathrm{H}(12 \mathrm{C})$ & 109.5 \\
$\mathrm{C}(11)-\mathrm{C}(13)-\mathrm{H}(13 \mathrm{~A})$ & 109.5 \\
$\mathrm{C}(11)-\mathrm{C}(13)-\mathrm{H}(13 \mathrm{~B})$ & 109.5 \\
$\mathrm{H}(13 \mathrm{~A})-\mathrm{C}(13)-\mathrm{H}(13 \mathrm{~B})$ & 109.5 \\
$\mathrm{C}(11)-\mathrm{C}(13)-\mathrm{H}(13 \mathrm{C})$ & 109.5 \\
$\mathrm{H}(13 \mathrm{~A})-\mathrm{C}(13)-\mathrm{H}(13 \mathrm{C})-\mathrm{C}(13)-\mathrm{H}(13 \mathrm{C})$ & 109.5 \\
\hline
\end{tabular}




\begin{tabular}{lc} 
& \\
\hline $\mathrm{C}(11)-\mathrm{C}(14)-\mathrm{H}(14 \mathrm{~A})$ & 109.5 \\
$\mathrm{C}(11)-\mathrm{C}(14)-\mathrm{H}(14 \mathrm{~B})$ & 109.5 \\
$\mathrm{H}(14 \mathrm{~A})-\mathrm{C}(14)-\mathrm{H}(14 \mathrm{~B})$ & 109.5 \\
$\mathrm{C}(11)-\mathrm{C}(14)-\mathrm{H}(14 \mathrm{C})$ & 109.5 \\
$\mathrm{H}(14 \mathrm{~A})-\mathrm{C}(14)-\mathrm{H}(14 \mathrm{C})$ & 109.5 \\
$\mathrm{H}(14 \mathrm{~B})-\mathrm{C}(14)-\mathrm{H}(14 \mathrm{C})$ & 109.5 \\
$\mathrm{C}(16)-\mathrm{C}(15)-\mathrm{S}(1)$ & $113.48(14)$ \\
$\mathrm{C}(16)-\mathrm{C}(15)-\mathrm{H}(15 \mathrm{~A})$ & 108.9 \\
$\mathrm{~S}(1)-\mathrm{C}(15)-\mathrm{H}(15 \mathrm{~A})$ & 108.9 \\
$\mathrm{C}(16)-\mathrm{C}(15)-\mathrm{H}(15 \mathrm{~B})$ & 108.9 \\
$\mathrm{~S}(1)-\mathrm{C}(15)-\mathrm{H}(15 \mathrm{~B})$ & 108.9 \\
$\mathrm{H}(15 \mathrm{~A})-\mathrm{C}(15)-\mathrm{H}(15 \mathrm{~B})$ & 107.7 \\
$\mathrm{C}(15)-\mathrm{C}(16)-\mathrm{S}(2)$ & $115.38(14)$ \\
$\mathrm{C}(15)-\mathrm{C}(16)-\mathrm{H}(16 \mathrm{~A})$ & 108.4 \\
$\mathrm{~S}(2)-\mathrm{C}(16)-\mathrm{H}(16 \mathrm{~A})$ & 108.4 \\
$\mathrm{C}(15)-\mathrm{C}(16)-\mathrm{H}(16 \mathrm{~B})$ & 108.4 \\
$\mathrm{~S}(2)-\mathrm{C}(16)-\mathrm{H}(16 \mathrm{~B})$ & 108.4 \\
$\mathrm{H}(16 \mathrm{~A})-\mathrm{C}(16)-\mathrm{H}(16 \mathrm{~B})$ & 107.5 \\
$\mathrm{C}(22)-\mathrm{C}(17)-\mathrm{C}(18)$ & $121.74(17)$ \\
$\mathrm{C}(22)-\mathrm{C}(17)-\mathrm{S}(2)$ & $122.27(14)$ \\
$\mathrm{C}(18)-\mathrm{C}(17)-\mathrm{S}(2)$ & $115.90(14)$ \\
$\mathrm{O}(2)-\mathrm{C}(18)-\mathrm{C}(17)$ & $118.34(16)$ \\
$\mathrm{O}(2)-\mathrm{C}(18)-\mathrm{C}(19)$ & $121.91(16)$ \\
$\mathrm{C}(17)-\mathrm{C}(18)-\mathrm{C}(19)$ & $119.74(16)$ \\
$\mathrm{C}(20)-\mathrm{C}(19)-\mathrm{C}(18)$ & $116.56(17)$ \\
$\mathrm{C}(20)-\mathrm{C}(19)-\mathrm{C}(23)$ & $122.44(16)$ \\
$\mathrm{C}(18)-\mathrm{C}(19)-\mathrm{C}(23)$ & $120.94(16)$ \\
$\mathrm{C}(19)-\mathrm{C}(20)-\mathrm{C}(21)$ & $124.69(17)$ \\
$\mathrm{C}(19)-\mathrm{C}(20)-\mathrm{H}(20)$ & 117.7 \\
$\mathrm{C}(21)-\mathrm{C}(20)-\mathrm{H}(20)$ & 117.7 \\
$\mathrm{C}(22)-\mathrm{C}(21)-\mathrm{C}(20)$ & $117.19(17)$ \\
$\mathrm{C}(22)-\mathrm{C}(21)-\mathrm{C}(27)$ & $122.76(17)$ \\
$\mathrm{C}(20)-\mathrm{C}(21)-\mathrm{C}(27)$ & $120.03(17)$ \\
$\mathrm{C}(21)-\mathrm{C}(22)-\mathrm{C}(17)$ & $120.01(17)$ \\
$\mathrm{C}(21)-\mathrm{C}(22)-\mathrm{H}(22)$ & 120.0 \\
$\mathrm{C}(17)-\mathrm{C}(22)-\mathrm{H}(22)$ & 120.0 \\
$\mathrm{C}(26)-\mathrm{C}(23)-\mathrm{C}(24)$ & $107.64(17)$ \\
$\mathrm{C}(26)-\mathrm{C}(23)-\mathrm{C}(25)$ & $107.53(17)$ \\
$\mathrm{C}(24)-\mathrm{C}(23)-\mathrm{C}(25)$ & $109.49(17)$ \\
$\mathrm{C}(26)-\mathrm{C}(23)-\mathrm{C}(19)$ & $111.84(16)$ \\
$\mathrm{C}(24)-\mathrm{C}(23)-\mathrm{C}(19)$ & $108.63(16)$ \\
$\mathrm{C}(25)-\mathrm{C}(23)-\mathrm{C}(19)$ & $111.63(16)$ \\
$\mathrm{C}(23)-\mathrm{C}(24)-\mathrm{H}(24 \mathrm{~A})$ & 109.5 \\
\hline
\end{tabular}

Symmetry transformations used to generate equivalent atoms: \#1 -x+1,-y,-z 
Table S4. Anisotropic displacement parameters $\left(\AA^{2} \times 10^{3}\right)$ for $\mathbf{2 b}$. The anisotropic displacement factor exponent takes the form: $-2 \pi^{2}\left[h^{2} a^{* 2} U 11+\ldots+2 h k a^{*} b^{*} U 12\right]$

\begin{tabular}{|c|c|c|c|c|c|c|}
\hline & U11 & $\mathrm{U} 22$ & U33 & $\mathrm{U} 23$ & U13 & $\mathrm{U} 12$ \\
\hline $\mathrm{Ti}$ & $16(1)$ & 12(1) & $22(1)$ & $6(1)$ & $3(1)$ & $(1)$ \\
\hline$S(1)$ & $24(1)$ & $15(1)$ & $28(1)$ & $5(1)$ & $9(1)$ & $8(1)$ \\
\hline$S(2)$ & $20(1)$ & 13(1) & $24(1)$ & $4(1)$ & $0(1)$ & $4(1)$ \\
\hline $\mathrm{O}(1)$ & $19(1)$ & $14(1)$ & $24(1)$ & $8(1)$ & $7(1)$ & $5(1)$ \\
\hline $\mathrm{O}(2)$ & $18(1)$ & $14(1)$ & $22(1)$ & $5(1)$ & $1(1)$ & $4(1)$ \\
\hline $\mathrm{C}(1)$ & 17(1) & $15(1)$ & $17(1)$ & $6(1)$ & $1(1)$ & $2(1)$ \\
\hline $\mathrm{C}(2)$ & 21(1) & $14(1)$ & $20(1)$ & $5(1)$ & $3(1)$ & $6(1)$ \\
\hline$C(3)$ & $22(1)$ & $23(1)$ & $21(1)$ & $9(1)$ & $8(1)$ & $8(1)$ \\
\hline C(4) & $23(1)$ & $19(1)$ & $23(1)$ & $10(1)$ & $5(1)$ & $4(1)$ \\
\hline$C(5)$ & $23(1)$ & $16(1)$ & $22(1)$ & $7(1)$ & $4(1)$ & $6(1)$ \\
\hline$C(6)$ & $18(1)$ & $15(1)$ & $17(1)$ & $4(1)$ & $2(1)$ & $4(1)$ \\
\hline $\mathrm{C}(7)$ & $30(1)$ & $25(1)$ & $39(1)$ & $19(1)$ & $16(1)$ & $9(1)$ \\
\hline $\mathrm{C}(8 \mathrm{~A})$ & $40(2)$ & $48(2)$ & $44(2)$ & $30(2)$ & $24(2)$ & $18(2)$ \\
\hline $\mathrm{C}(9 \mathrm{~A})$ & $25(2)$ & $44(2)$ & $59(2)$ & $31(2)$ & $11(2)$ & $5(2)$ \\
\hline$C(10 A)$ & $46(2)$ & $26(2)$ & $63(2)$ & $25(2)$ & $31(2)$ & $10(1)$ \\
\hline $\mathrm{C}(8 \mathrm{~B})$ & $54(9)$ & $48(9)$ & $89(12)$ & $45(9)$ & $39(9)$ & 19(7) \\
\hline $\mathrm{C}(9 \mathrm{~B})$ & $50(9)$ & $56(10)$ & $106(14)$ & $67(11)$ & $45(10$ & 0) $19(8)$ \\
\hline $\mathrm{C}(10 \mathrm{~B})$ & ) 98(13) & $41(8)$ & $52(9)$ & $-4(7)$ & $45(10)$ & $-42(9)$ \\
\hline $\mathrm{C}(11)$ & $24(1)$ & $15(1)$ & $25(1)$ & $6(1)$ & $9(1)$ & $7(1)$ \\
\hline$C(12)$ & 21(1) & 21(1) & $31(1)$ & $6(1)$ & $7(1)$ & $9(1)$ \\
\hline$C(13)$ & $32(1)$ & $29(1)$ & $24(1)$ & $4(1)$ & $10(1)$ & $7(1)$ \\
\hline $\mathrm{C}(14)$ & $33(1)$ & $19(1)$ & $46(1)$ & $8(1)$ & $17(1)$ & $12(1)$ \\
\hline$C(15)$ & 21(1) & $22(1)$ & $44(1)$ & $16(1)$ & 11(1) & $10(1)$ \\
\hline$C(16)$ & $18(1)$ & $18(1)$ & $41(1)$ & $13(1)$ & $2(1)$ & $2(1)$ \\
\hline$C(17)$ & $22(1)$ & 11(1) & $22(1)$ & $6(1)$ & $6(1)$ & $6(1)$ \\
\hline $\mathrm{C}(18)$ & $18(1)$ & $17(1)$ & $19(1)$ & $7(1)$ & $5(1)$ & $6(1)$ \\
\hline$C(19)$ & $21(1)$ & $15(1)$ & $19(1)$ & $6(1)$ & $7(1)$ & $5(1)$ \\
\hline$C(20)$ & $26(1)$ & $16(1)$ & $23(1)$ & $9(1)$ & $10(1)$ & $10(1)$ \\
\hline $\mathrm{C}(21)$ & $19(1)$ & $20(1)$ & $21(1)$ & $9(1)$ & $7(1)$ & $8(1)$ \\
\hline $\mathrm{C}(22)$ & $18(1)$ & $19(1)$ & $19(1)$ & $6(1)$ & $3(1)$ & $4(1)$ \\
\hline $\mathrm{C}(23)$ & $24(1)$ & $12(1)$ & $25(1)$ & $5(1)$ & $4(1)$ & $5(1)$ \\
\hline$C(24)$ & $24(1)$ & $23(1)$ & $39(1)$ & $8(1)$ & $7(1)$ & $-1(1)$ \\
\hline$C(25)$ & $37(1)$ & 21(1) & $23(1)$ & $2(1)$ & $2(1)$ & $6(1)$ \\
\hline$C(26)$ & $38(1)$ & $16(1)$ & $41(1)$ & $4(1)$ & $-2(1)$ & $7(1)$ \\
\hline $\mathrm{C}(27)$ & $22(1)$ & $23(1)$ & $27(1)$ & $12(1)$ & $8(1)$ & $11(1)$ \\
\hline$C(28)$ & $25(1)$ & $33(1)$ & $34(1)$ & $13(1)$ & $11(1)$ & $14(1)$ \\
\hline$C(29)$ & $30(1)$ & $38(1)$ & $39(1)$ & $26(1)$ & $10(1)$ & $17(1)$ \\
\hline $\mathrm{C}(30)$ & $28(1)$ & $34(1)$ & $29(1)$ & $11(1)$ & $1(1)$ & $16(1)$ \\
\hline $\mathrm{C}(31)$ & $23(1)$ & $18(1)$ & $23(1)$ & $4(1)$ & $3(1)$ & $6(1)$ \\
\hline $\mathrm{C}(32)$ & $23(1)$ & $20(1)$ & $17(1)$ & $3(1)$ & $3(1)$ & $7(1)$ \\
\hline $\mathrm{C}(33)$ & $23(1)$ & $24(1)$ & $25(1)$ & $7(1)$ & $6(1)$ & $4(1)$ \\
\hline $\mathrm{C}(34)$ & $33(1)$ & $23(1)$ & $29(1)$ & $13(1)$ & $8(1)$ & $5(1)$ \\
\hline$C(35)$ & $34(1)$ & $27(1)$ & $30(1)$ & $12(1)$ & $4(1)$ & $14(1)$ \\
\hline$C(36)$ & $26(1)$ & $33(1)$ & $33(1)$ & $12(1)$ & $9(1)$ & $13(1)$ \\
\hline$C(37)$ & $24(1)$ & $27(1)$ & $24(1)$ & $10(1)$ & $7(1)$ & $5(1)$ \\
\hline $\mathrm{C}(38)$ & $21(1)$ & $19(1)$ & $29(1)$ & $11(1)$ & $6(1)$ & $5(1)$ \\
\hline
\end{tabular}




\begin{tabular}{llllccc}
\hline $\mathrm{C}(39)$ & $19(1)$ & $24(1)$ & $31(1)$ & $12(1)$ & $12(1)$ & $2(1)$ \\
$\mathrm{C}(40)$ & $29(1)$ & $29(1)$ & $33(1)$ & $14(1)$ & $12(1)$ & $7(1)$ \\
$\mathrm{C}(41)$ & $32(1)$ & $44(2)$ & $37(1)$ & $24(1)$ & $5(1)$ & $6(1)$ \\
$\mathrm{C}(42)$ & $42(1)$ & $39(1)$ & $31(1)$ & $10(1)$ & $2(1)$ & $-4(1)$ \\
$\mathrm{C}(43)$ & $43(1)$ & $26(1)$ & $36(1)$ & $5(1)$ & $10(1)$ & $-1(1)$ \\
$\mathrm{C}(44)$ & $27(1)$ & $24(1)$ & $35(1)$ & $11(1)$ & $9(1)$ & $4(1)$ \\
$\mathrm{C}(45)$ & $43(4)$ & $47(4)$ & $54(4)$ & $28(3)$ & $7(3)$ & $-9(3)$ \\
$\mathrm{C}(46)$ & $75(10)$ & $44(5)$ & $35(7)$ & $-8(6)$ & $23(6)$ & $-14(6)$ \\
$\mathrm{C}(47)$ & $78(5)$ & $40(3)$ & $62(4)$ & $15(3)$ & $39(4)$ & $19(3)$ \\
$\mathrm{C}(48)$ & $66(6)$ & $39(5)$ & $50(5)$ & $25(4)$ & $28(5)$ & $25(5)$ \\
$\mathrm{C}(49)$ & $40(3)$ & $25(3)$ & $44(3)$ & $5(2)$ & $14(2)$ & $3(2)$ \\
$\mathrm{C}(50)$ & $39(5)$ & $27(4)$ & $55(9)$ & $2(6)$ & $19(6)$ & $4(4)$ \\
$\mathrm{C}(51)$ & $74(9)$ & $104(11)$ & $95(12)$ & $62(9)$ & $-11(8)$ & $-22(8)$ \\
\hline
\end{tabular}


Table S5. Hydrogen coordinates $\left(\times 10^{4}\right)$ and isotropic displacement parameters $\left(\AA^{2} \times 10^{3}\right)$ for $\mathbf{2 b}$.

\begin{tabular}{|c|c|c|c|c|}
\hline & $\mathrm{x}$ & $\mathrm{y}$ & $\mathrm{Z}$ & $\mathrm{U}(\mathrm{eq})$ \\
\hline $\mathrm{H}(3)$ & 6133 & 4757 & 1455 & 25 \\
\hline $\mathrm{H}(5)$ & 4114 & 6632 & 2420 & 25 \\
\hline $\mathrm{H}(8 \mathrm{~A})$ & 4910 & 6117 & 360 & 57 \\
\hline $\mathrm{H}(8 \mathrm{~B})$ & 6296 & 5680 & 432 & 57 \\
\hline $\mathrm{H}(8 \mathrm{C})$ & 6581 & 6730 & 448 & 57 \\
\hline $\mathrm{H}(9 \mathrm{~A})$ & 8214 & 6053 & 1892 & 62 \\
\hline $\mathrm{H}(9 \mathrm{~B})$ & 8298 & 6887 & 2719 & 62 \\
\hline $\mathrm{H}(9 \mathrm{C})$ & 8687 & 7090 & 1866 & 62 \\
\hline $\mathrm{H}(10 \mathrm{~A})$ & 6802 & 7950 & 1766 & 60 \\
\hline $\mathrm{H}(10 \mathrm{~B})$ & 6263 & 7718 & 2572 & 60 \\
\hline $\mathrm{H}(10 \mathrm{C})$ & 5041 & 7444 & 1619 & 60 \\
\hline $\mathrm{H}(8 \mathrm{D})$ & 6345 & 7346 & 921 & 83 \\
\hline $\mathrm{H}(8 \mathrm{E})$ & 4822 & 7075 & 1203 & 83 \\
\hline $\mathrm{H}(8 \mathrm{~F})$ & 5164 & 6330 & 464 & 83 \\
\hline $\mathrm{H}(9 \mathrm{D})$ & 7385 & 5774 & 818 & 88 \\
\hline $\mathrm{H}(9 \mathrm{E})$ & 8203 & 5954 & 1844 & 88 \\
\hline $\mathrm{H}(9 \mathrm{~F})$ & 8555 & 6764 & 1416 & 88 \\
\hline $\mathrm{H}(10 \mathrm{D})$ & 7982 & 7915 & 2434 & 112 \\
\hline $\mathrm{H}(10 \mathrm{E})$ & 7877 & 7231 & 3016 & 112 \\
\hline $\mathrm{H}(10 \mathrm{~F})$ & 6544 & 7692 & 2801 & 112 \\
\hline $\mathrm{H}(12 \mathrm{~A})$ & -314 & 5187 & 2819 & 36 \\
\hline $\mathrm{H}(12 \mathrm{~B})$ & 386 & 4370 & 2493 & 36 \\
\hline $\mathrm{H}(12 \mathrm{C})$ & 109 & 5030 & 1906 & 36 \\
\hline $\mathrm{H}(13 \mathrm{~A})$ & 3540 & 6085 & 4294 & 44 \\
\hline $\mathrm{H}(13 \mathrm{~B})$ & 2583 & 5016 & 3966 & 44 \\
\hline $\mathrm{H}(13 \mathrm{C})$ & 1787 & 5788 & 4276 & 44 \\
\hline $\mathrm{H}(14 \mathrm{~A})$ & 1686 & 6683 & 2453 & 48 \\
\hline $\mathrm{H}(14 \mathrm{~B})$ & 2997 & 7086 & 3386 & 48 \\
\hline $\mathrm{H}(14 \mathrm{C})$ & 1237 & 6796 & 3357 & 48 \\
\hline $\mathrm{H}(15 \mathrm{~A})$ & 6353 & 2556 & 2416 & 33 \\
\hline $\mathrm{H}(15 \mathrm{~B})$ & 7111 & 3559 & 2372 & 33 \\
\hline $\mathrm{H}(16 \mathrm{~A})$ & 6318 & 4256 & 3544 & 32 \\
\hline $\mathrm{H}(16 \mathrm{~B})$ & 7258 & 3599 & 3841 & 32 \\
\hline $\mathrm{H}(20)$ & 4824 & -542 & 3115 & 24 \\
\hline $\mathrm{H}(22)$ & 6856 & 2151 & 4529 & 23 \\
\hline $\mathrm{H}(24 \mathrm{~A})$ & -136 & -1007 & 2008 & 46 \\
\hline $\mathrm{H}(24 \mathrm{~B})$ & 545 & 90 & 2438 & 46 \\
\hline $\mathrm{H}(24 \mathrm{C})$ & 963 & -564 & 3007 & 46 \\
\hline$H(25 A)$ & 2685 & -476 & 907 & 44 \\
\hline $\mathrm{H}(25 \mathrm{~B})$ & 1703 & 198 & 1178 & 44 \\
\hline $\mathrm{H}(25 \mathrm{C})$ & 889 & -890 & 746 & 44 \\
\hline $\mathrm{H}(26 \mathrm{~A})$ & 2534 & -1581 & 2566 & 53 \\
\hline $\mathrm{H}(26 \mathrm{~B})$ & 3263 & -1512 & 1777 & 53 \\
\hline $\mathrm{H}(26 \mathrm{C})$ & 1459 & -1954 & 1561 & 53 \\
\hline $\mathrm{H}(28 \mathrm{~A})$ & 8650 & 668 & 3506 & 43 \\
\hline $\mathrm{H}(28 \mathrm{~B})$ & 7578 & -370 & 3237 & 43 \\
\hline
\end{tabular}




\begin{tabular}{lcccc}
\hline $\mathrm{H}(28 \mathrm{C})$ & 9138 & -7 & 4033 & 43 \\
$\mathrm{H}(29 \mathrm{~A})$ & 7678 & -495 & 5035 & 48 \\
$\mathrm{H}(29 \mathrm{~B})$ & 6122 & -853 & 4236 & 48 \\
$\mathrm{H}(29 \mathrm{C})$ & 6260 & -128 & 5158 & 48 \\
$\mathrm{H}(30 \mathrm{~A})$ & 9361 & 1093 & 5478 & 45 \\
$\mathrm{H}(30 \mathrm{~B})$ & 7978 & 1490 & 5614 & 45 \\
$\mathrm{H}(30 \mathrm{C})$ & 8932 & 1804 & 4985 & 45 \\
$\mathrm{H}(31 \mathrm{~A})$ & 1425 & 1853 & 712 & 27 \\
$\mathrm{H}(31 \mathrm{~B})$ & -34 & 1405 & 978 & 27 \\
$\mathrm{H}(33)$ & 1649 & 3314 & 348 & 30 \\
$\mathrm{H}(34)$ & 340 & 4213 & -257 & 34 \\
$\mathrm{H}(35)$ & -2203 & 4067 & -268 & 36 \\
$\mathrm{H}(36)$ & -3413 & 3006 & 337 & 35 \\
$\mathrm{H}(37)$ & -2126 & 2091 & 925 & 30 \\
$\mathrm{H}(38 \mathrm{~A})$ & -719 & 2607 & 2591 & 27 \\
$\mathrm{H}(38 \mathrm{~B})$ & -546 & 1615 & 2640 & 27 \\
$\mathrm{H}(40)$ & 1650 & 1668 & 3997 & 35 \\
$\mathrm{H}(41)$ & 2971 & 2390 & 5497 & 45 \\
$\mathrm{H}(42)$ & 2808 & 3840 & 6237 & 50 \\
$\mathrm{H}(43)$ & 1291 & 4545 & 5458 & 46 \\
$\mathrm{H}(44)$ & 45 & 3858 & 3952 & 35 \\
$\mathrm{H}(46)$ & 5125 & 1027 & 979 & 72 \\
$\mathrm{H}(47)$ & 3465 & 1358 & -144 & 68 \\
$\mathrm{H}(48)$ & 2881 & 425 & -1592 & 55 \\
$\mathrm{H}(49)$ & 3622 & -901 & -1919 & 47 \\
$\mathrm{H}(50)$ & 5228 & -1231 & -793 & 51 \\
$\mathrm{H}(51 \mathrm{~A})$ & 6342 & -901 & 868 & 150 \\
$\mathrm{H}(51 \mathrm{~B})$ & 6360 & 49 & 1529 & 150 \\
$\mathrm{H}(51 \mathrm{C})$ & 7557 & 46 & 969 & 150 \\
\hline & & & &
\end{tabular}


Table S6. Torsion angles $\left[{ }^{\circ}\right]$ for $\mathbf{2 b}$.

\begin{tabular}{|c|c|}
\hline $\mathrm{O}(1)-\mathrm{Ti}-\mathrm{S}(1)-\mathrm{C}(2)$ & $-0.75(7)$ \\
\hline $\mathrm{O}(2)-\mathrm{Ti}-\mathrm{S}(1)-\mathrm{C}(2)$ & $-163.92(7)$ \\
\hline $\mathrm{C}(38)-\mathrm{Ti}-\mathrm{S}(1)-\mathrm{C}(2)$ & $54.1(3)$ \\
\hline $\mathrm{C}(31)-\mathrm{Ti}-\mathrm{S}(1)-\mathrm{C}(2)$ & $103.24(8)$ \\
\hline $\mathrm{S}(2)-\mathrm{Ti}-\mathrm{S}(1)-\mathrm{C}(2)$ & $-91.67(6)$ \\
\hline $\mathrm{O}(1)-\mathrm{Ti}-\mathrm{S}(1)-\mathrm{C}(15)$ & $102.32(8)$ \\
\hline $\mathrm{O}(2)-\mathrm{Ti}-\mathrm{S}(1)-\mathrm{C}(15)$ & $-60.84(8)$ \\
\hline $\mathrm{C}(38)-\mathrm{Ti}-\mathrm{S}(1)-\mathrm{C}(15)$ & $157.2(3)$ \\
\hline $\mathrm{C}(31)-\mathrm{Ti}-\mathrm{S}(1)-\mathrm{C}(15)$ & $-153.68(9)$ \\
\hline S(2)-Ti-S(1)-C(15) & $11.41(7)$ \\
\hline $\mathrm{O}(1)-\mathrm{Ti}-\mathrm{S}(2)-\mathrm{C}(17)$ & $-166.10(8)$ \\
\hline $\mathrm{O}(2)-\mathrm{Ti}-\mathrm{S}(2)-\mathrm{C}(17)$ & $4.10(8)$ \\
\hline $\mathrm{C}(38)-\mathrm{Ti}-\mathrm{S}(2)-\mathrm{C}(17)$ & $93.84(9)$ \\
\hline $\mathrm{C}(31)-\mathrm{Ti}-\mathrm{S}(2)-\mathrm{C}(17)$ & $-61.03(13)$ \\
\hline $\mathrm{S}(1)-\mathrm{Ti}-\mathrm{S}(2)-\mathrm{C}(17)$ & $-93.47(7)$ \\
\hline $\mathrm{O}(1)-\mathrm{Ti}-\mathrm{S}(2)-\mathrm{C}(16)$ & $-61.15(8)$ \\
\hline $\mathrm{O}(2)-\mathrm{Ti}-\mathrm{S}(2)-\mathrm{C}(16)$ & $109.05(8)$ \\
\hline $\mathrm{C}(38)-\mathrm{Ti}-\mathrm{S}(2)-\mathrm{C}(16)$ & $-161.20(9)$ \\
\hline $\mathrm{C}(31)-\mathrm{Ti}-\mathrm{S}(2)-\mathrm{C}(16)$ & 43.93(14) \\
\hline $\mathrm{S}(1)-\mathrm{Ti}-\mathrm{S}(2)-\mathrm{C}(16)$ & $11.49(7)$ \\
\hline $\mathrm{O}(2)-\mathrm{Ti}-\mathrm{O}(1)-\mathrm{C}(1)$ & $53.0(3)$ \\
\hline $\mathrm{C}(38)-\mathrm{Ti}-\mathrm{O}(1)-\mathrm{C}(1)$ & $-166.53(17)$ \\
\hline $\mathrm{C}(31)-\mathrm{Ti}-\mathrm{O}(1)-\mathrm{C}(1)$ & $-73.91(18)$ \\
\hline $\mathrm{S}(2)-\mathrm{Ti}-\mathrm{O}(1)-\mathrm{C}(1)$ & $78.30(17)$ \\
\hline $\mathrm{S}(1)-\mathrm{Ti}-\mathrm{O}(1)-\mathrm{C}(1)$ & $3.69(16)$ \\
\hline $\mathrm{O}(1)-\mathrm{Ti}-\mathrm{O}(2)-\mathrm{C}(18)$ & $21.2(3)$ \\
\hline $\mathrm{C}(38)-\mathrm{Ti}-\mathrm{O}(2)-\mathrm{C}(18)$ & $-119.84(18)$ \\
\hline $\mathrm{C}(31)-\mathrm{Ti}-\mathrm{O}(2)-\mathrm{C}(18)$ & $149.21(18)$ \\
\hline $\mathrm{S}(2)-\mathrm{Ti}-\mathrm{O}(2)-\mathrm{C}(18)$ & $-5.23(16)$ \\
\hline $\mathrm{S}(1)-\mathrm{Ti}-\mathrm{O}(2)-\mathrm{C}(18)$ & $67.42(17)$ \\
\hline $\mathrm{Ti}-\mathrm{O}(1)-\mathrm{C}(1)-\mathrm{C}(2)$ & $-5.7(3)$ \\
\hline $\mathrm{Ti}-\mathrm{O}(1)-\mathrm{C}(1)-\mathrm{C}(6)$ & $172.92(13)$ \\
\hline $\mathrm{O}(1)-\mathrm{C}(1)-\mathrm{C}(2)-\mathrm{C}(3)$ & $-179.27(16)$ \\
\hline $\mathrm{C}(6)-\mathrm{C}(1)-\mathrm{C}(2)-\mathrm{C}(3)$ & $2.1(3)$ \\
\hline $\mathrm{O}(1)-\mathrm{C}(1)-\mathrm{C}(2)-\mathrm{S}(1)$ & $3.4(2)$ \\
\hline $\mathrm{C}(6)-\mathrm{C}(1)-\mathrm{C}(2)-\mathrm{S}(1)$ & $-175.27(13)$ \\
\hline$C(15)-S(1)-C(2)-C(3)$ & 74.39(17) \\
\hline $\mathrm{Ti}-\mathrm{S}(1)-\mathrm{C}(2)-\mathrm{C}(3)$ & $-178.46(15)$ \\
\hline$C(15)-S(1)-C(2)-C(1)$ & $-108.28(16)$ \\
\hline $\mathrm{Ti}-\mathrm{S}(1)-\mathrm{C}(2)-\mathrm{C}(1)$ & $-1.13(15)$ \\
\hline$C(1)-C(2)-C(3)-C(4)$ & $-0.3(3)$ \\
\hline $\mathrm{S}(1)-\mathrm{C}(2)-\mathrm{C}(3)-\mathrm{C}(4)$ & $176.97(15)$ \\
\hline$C(2)-C(3)-C(4)-C(5)$ & $-1.7(3)$ \\
\hline $\mathrm{C}(2)-\mathrm{C}(3)-\mathrm{C}(4)-\mathrm{C}(7)$ & $-179.62(18)$ \\
\hline$C(3)-C(4)-C(5)-C(6)$ & $1.9(3)$ \\
\hline $\mathrm{C}(7)-\mathrm{C}(4)-\mathrm{C}(5)-\mathrm{C}(6)$ & $179.84(18)$ \\
\hline $\mathrm{C}(4)-\mathrm{C}(5)-\mathrm{C}(6)-\mathrm{C}(1)$ & $-0.1(3)$ \\
\hline $\mathrm{C}(4)-\mathrm{C}(5)-\mathrm{C}(6)-\mathrm{C}(11)$ & $177.78(18)$ \\
\hline
\end{tabular}




\begin{tabular}{|c|c|}
\hline $\mathrm{O}(1)-\mathrm{C}(1)-\mathrm{C}(6)-\mathrm{C}(5)$ & $179.54(16)$ \\
\hline $\mathrm{C}(2)-\mathrm{C}(1)-\mathrm{C}(6)-\mathrm{C}(5)$ & $-1.8(3)$ \\
\hline $\mathrm{O}(1)-\mathrm{C}(1)-\mathrm{C}(6)-\mathrm{C}(11)$ & $1.6(3)$ \\
\hline$C(2)-C(1)-C(6)-C(11)$ & $-179.77(17)$ \\
\hline $\mathrm{C}(3)-\mathrm{C}(4)-\mathrm{C}(7)-\mathrm{C}(8 \mathrm{~B})$ & $113.4(8)$ \\
\hline $\mathrm{C}(5)-\mathrm{C}(4)-\mathrm{C}(7)-\mathrm{C}(8 \mathrm{~B})$ & $-64.5(8)$ \\
\hline$C(3)-C(4)-C(7)-C(9 A)$ & $-56.1(3)$ \\
\hline$C(5)-C(4)-C(7)-C(9 A)$ & $126.0(3)$ \\
\hline$C(3)-C(4)-C(7)-C(10 A)$ & $-179.8(2)$ \\
\hline$C(5)-C(4)-C(7)-C(10 A)$ & $2.4(3)$ \\
\hline$C(3)-C(4)-C(7)-C(8 A)$ & $63.4(3)$ \\
\hline$C(5)-C(4)-C(7)-C(8 A)$ & $-114.4(2)$ \\
\hline$C(3)-C(4)-C(7)-C(9 B)$ & $-13.6(9)$ \\
\hline $\mathrm{C}(5)-\mathrm{C}(4)-\mathrm{C}(7)-\mathrm{C}(9 \mathrm{~B})$ & $168.6(8)$ \\
\hline $\mathrm{C}(3)-\mathrm{C}(4)-\mathrm{C}(7)-\mathrm{C}(10 \mathrm{~B})$ & $-124.7(8)$ \\
\hline$C(5)-C(4)-C(7)-C(10 B)$ & $57.5(9)$ \\
\hline$C(5)-C(6)-C(11)-C(14)$ & $7.1(3)$ \\
\hline$C(1)-C(6)-C(11)-C(14)$ & $-175.10(17)$ \\
\hline$C(5)-C(6)-C(11)-C(13)$ & $-111.6(2)$ \\
\hline$C(1)-C(6)-C(11)-C(13)$ & $66.3(2)$ \\
\hline$C(5)-C(6)-C(11)-C(12)$ & $126.84(19)$ \\
\hline$C(1)-C(6)-C(11)-C(12)$ & $-55.3(2)$ \\
\hline$C(2)-S(1)-C(15)-C(16)$ & $56.56(16)$ \\
\hline Ti-S(1)-C(15)-C(16) & $-39.39(15)$ \\
\hline$S(1)-C(15)-C(16)-S(2)$ & $55.52(18)$ \\
\hline$C(17)-S(2)-C(16)-C(15)$ & $58.47(16)$ \\
\hline $\mathrm{Ti}-\mathrm{S}(2)-\mathrm{C}(16)-\mathrm{C}(15)$ & $-41.00(16)$ \\
\hline $\mathrm{C}(16)-\mathrm{S}(2)-\mathrm{C}(17)-\mathrm{C}(22)$ & $68.28(18)$ \\
\hline $\mathrm{Ti}-\mathrm{S}(2)-\mathrm{C}(17)-\mathrm{C}(22)$ & $179.11(16)$ \\
\hline $\mathrm{C}(16)-\mathrm{S}(2)-\mathrm{C}(17)-\mathrm{C}(18)$ & $-115.12(16)$ \\
\hline $\mathrm{Ti}-\mathrm{S}(2)-\mathrm{C}(17)-\mathrm{C}(18)$ & $-4.29(15)$ \\
\hline $\mathrm{Ti}-\mathrm{O}(2)-\mathrm{C}(18)-\mathrm{C}(17)$ & $3.8(3)$ \\
\hline $\mathrm{Ti}-\mathrm{O}(2)-\mathrm{C}(18)-\mathrm{C}(19)$ & $-177.83(13)$ \\
\hline $\mathrm{C}(22)-\mathrm{C}(17)-\mathrm{C}(18)-\mathrm{O}(2)$ & 178.94(17) \\
\hline $\mathrm{S}(2)-\mathrm{C}(17)-\mathrm{C}(18)-\mathrm{O}(2)$ & $2.3(2)$ \\
\hline$C(22)-C(17)-C(18)-C(19)$ & $0.5(3)$ \\
\hline$S(2)-C(17)-C(18)-C(19)$ & $-176.14(14)$ \\
\hline $\mathrm{O}(2)-\mathrm{C}(18)-\mathrm{C}(19)-\mathrm{C}(20)$ & 179.19(16) \\
\hline$C(17)-C(18)-C(19)-C(20)$ & $-2.4(3)$ \\
\hline $\mathrm{O}(2)-\mathrm{C}(18)-\mathrm{C}(19)-\mathrm{C}(23)$ & $-3.5(3)$ \\
\hline $\mathrm{C}(17)-\mathrm{C}(18)-\mathrm{C}(19)-\mathrm{C}(23)$ & $174.94(17)$ \\
\hline$C(18)-C(19)-C(20)-C(21)$ & $2.3(3)$ \\
\hline $\mathrm{C}(23)-\mathrm{C}(19)-\mathrm{C}(20)-\mathrm{C}(21)$ & $-174.99(18)$ \\
\hline$C(19)-C(20)-C(21)-C(22)$ & $-0.2(3)$ \\
\hline$C(19)-C(20)-C(21)-C(27)$ & $-179.11(17)$ \\
\hline$C(20)-C(21)-C(22)-C(17)$ & $-1.9(3)$ \\
\hline $\mathrm{C}(27)-\mathrm{C}(21)-\mathrm{C}(22)-\mathrm{C}(17)$ & $177.01(17)$ \\
\hline$C(18)-C(17)-C(22)-C(21)$ & $1.8(3)$ \\
\hline$S(2)-C(17)-C(22)-C(21)$ & $178.19(14)$ \\
\hline$C(20)-C(19)-C(23)-C(26)$ & $-3.7(3)$ \\
\hline$C(18)-C(19)-C(23)-C(26)$ & $179.15(18)$ \\
\hline
\end{tabular}




\begin{tabular}{|c|c|}
\hline $\mathrm{C}(20)-\mathrm{C}(19)-\mathrm{C}(23)-\mathrm{C}(24)$ & $115.0(2)$ \\
\hline $\mathrm{C}(18)-\mathrm{C}(19)-\mathrm{C}(23)-\mathrm{C}(24)$ & $-62.2(2)$ \\
\hline$C(20)-C(19)-C(23)-C(25)$ & $-124.2(2)$ \\
\hline $\mathrm{C}(18)-\mathrm{C}(19)-\mathrm{C}(23)-\mathrm{C}(25)$ & $58.6(2)$ \\
\hline$C(22)-C(21)-C(27)-C(30)$ & $10.6(3)$ \\
\hline$C(20)-C(21)-C(27)-C(30)$ & $-170.48(18)$ \\
\hline $\mathrm{C}(22)-\mathrm{C}(21)-\mathrm{C}(27)-\mathrm{C}(28)$ & $-109.6(2)$ \\
\hline $\mathrm{C}(20)-\mathrm{C}(21)-\mathrm{C}(27)-\mathrm{C}(28)$ & $69.3(2)$ \\
\hline $\mathrm{C}(22)-\mathrm{C}(21)-\mathrm{C}(27)-\mathrm{C}(29)$ & $131.3(2)$ \\
\hline $\mathrm{C}(20)-\mathrm{C}(21)-\mathrm{C}(27)-\mathrm{C}(29)$ & $-49.8(2)$ \\
\hline $\mathrm{O}(1)-\mathrm{Ti}-\mathrm{C}(31)-\mathrm{C}(32)$ & $-25.34(16)$ \\
\hline $\mathrm{O}(2)-\mathrm{Ti}-\mathrm{C}(31)-\mathrm{C}(32)$ & $172.47(15)$ \\
\hline $\mathrm{C}(38)-\mathrm{Ti}-\mathrm{C}(31)-\mathrm{C}(32)$ & $75.73(15)$ \\
\hline $\mathrm{S}(2)-\mathrm{Ti}-\mathrm{C}(31)-\mathrm{C}(32)$ & $-126.83(13)$ \\
\hline $\mathrm{S}(1)-\mathrm{Ti}-\mathrm{C}(31)-\mathrm{C}(32)$ & $-95.39(15)$ \\
\hline $\mathrm{O}(1)-\mathrm{Ti}-\mathrm{C}(31)-\mathrm{C}(38)$ & $-101.07(7)$ \\
\hline $\mathrm{O}(2)-\mathrm{Ti}-\mathrm{C}(31)-\mathrm{C}(38)$ & $96.75(7)$ \\
\hline $\mathrm{S}(2)-\mathrm{Ti}-\mathrm{C}(31)-\mathrm{C}(38)$ & $157.45(12)$ \\
\hline S(1)-Ti-C(31)-C(38) & $-171.12(6)$ \\
\hline Ti-C(31)-C(32)-C(33) & $87.8(2)$ \\
\hline $\mathrm{C}(38)-\mathrm{C}(31)-\mathrm{C}(32)-\mathrm{C}(33)$ & $131.32(17)$ \\
\hline Ti-C(31)-C(32)-C(37) & $-92.3(2)$ \\
\hline $\mathrm{C}(38)-\mathrm{C}(31)-\mathrm{C}(32)-\mathrm{C}(37)$ & $-48.8(2)$ \\
\hline $\mathrm{C}(37)-\mathrm{C}(32)-\mathrm{C}(33)-\mathrm{C}(34)$ & $-1.1(3)$ \\
\hline $\mathrm{C}(31)-\mathrm{C}(32)-\mathrm{C}(33)-\mathrm{C}(34)$ & $178.79(19)$ \\
\hline $\mathrm{C}(32)-\mathrm{C}(33)-\mathrm{C}(34)-\mathrm{C}(35)$ & $0.8(3)$ \\
\hline $\mathrm{C}(33)-\mathrm{C}(34)-\mathrm{C}(35)-\mathrm{C}(36)$ & $0.1(3)$ \\
\hline $\mathrm{C}(34)-\mathrm{C}(35)-\mathrm{C}(36)-\mathrm{C}(37)$ & $-0.5(3)$ \\
\hline$C(35)-C(36)-C(37)-C(32)$ & $0.2(3)$ \\
\hline $\mathrm{C}(33)-\mathrm{C}(32)-\mathrm{C}(37)-\mathrm{C}(36)$ & $0.6(3)$ \\
\hline $\mathrm{C}(31)-\mathrm{C}(32)-\mathrm{C}(37)-\mathrm{C}(36)$ & $-179.27(19)$ \\
\hline $\mathrm{O}(1)-\mathrm{Ti}-\mathrm{C}(38)-\mathrm{C}(39)$ & $-76.49(12)$ \\
\hline $\mathrm{O}(2)-\mathrm{Ti}-\mathrm{C}(38)-\mathrm{C}(39)$ & $89.35(12)$ \\
\hline $\mathrm{C}(31)-\mathrm{Ti}-\mathrm{C}(38)-\mathrm{C}(39)$ & $-177.38(12)$ \\
\hline $\mathrm{S}(2)-\mathrm{Ti}-\mathrm{C}(38)-\mathrm{C}(39)$ & $14.25(13)$ \\
\hline $\mathrm{S}(1)-\mathrm{Ti}-\mathrm{C}(38)-\mathrm{C}(39)$ & $-128.9(2)$ \\
\hline $\mathrm{O}(1)-\mathrm{Ti}-\mathrm{C}(38)-\mathrm{C}(31)$ & $100.89(7)$ \\
\hline $\mathrm{O}(2)-\mathrm{Ti}-\mathrm{C}(38)-\mathrm{C}(31)$ & $-93.27(7)$ \\
\hline $\mathrm{S}(2)-\mathrm{Ti}-\mathrm{C}(38)-\mathrm{C}(31)$ & $-168.37(6)$ \\
\hline $\mathrm{S}(1)-\mathrm{Ti}-\mathrm{C}(38)-\mathrm{C}(31)$ & $48.5(3)$ \\
\hline $\mathrm{C}(32)-\mathrm{C}(31)-\mathrm{C}(38)-\mathrm{C}(39)$ & $-114.1(2)$ \\
\hline Ti-C (31)-C(38)-C(39) & $4.2(2)$ \\
\hline $\mathrm{C}(32)-\mathrm{C}(31)-\mathrm{C}(38)-\mathrm{Ti}$ & $-118.32(14)$ \\
\hline Ti-C(38)-C(39)-C(44) & $104.18(18)$ \\
\hline $\mathrm{C}(31)-\mathrm{C}(38)-\mathrm{C}(39)-\mathrm{C}(44)$ & $101.2(2)$ \\
\hline Ti-C (38)-C(39)-C(40) & $-71.30(19)$ \\
\hline $\mathrm{C}(31)-\mathrm{C}(38)-\mathrm{C}(39)-\mathrm{C}(40)$ & $-74.3(3)$ \\
\hline $\mathrm{C}(44)-\mathrm{C}(39)-\mathrm{C}(40)-\mathrm{C}(41)$ & $-1.5(3)$ \\
\hline $\mathrm{C}(38)-\mathrm{C}(39)-\mathrm{C}(40)-\mathrm{C}(41)$ & $174.2(2)$ \\
\hline $\mathrm{C}(39)-\mathrm{C}(40)-\mathrm{C}(41)-\mathrm{C}(42)$ & $1.3(3)$ \\
\hline$C(40)-C(41)-C(42)-C(43)$ & $0.3(4)$ \\
\hline
\end{tabular}




$\begin{array}{lc}\mathrm{C}(41)-\mathrm{C}(42)-\mathrm{C}(43)-\mathrm{C}(44) & -1.6(4) \\ \mathrm{C}(42)-\mathrm{C}(43)-\mathrm{C}(44)-\mathrm{C}(39) & 1.3(3) \\ \mathrm{C}(40)-\mathrm{C}(39)-\mathrm{C}(44)-\mathrm{C}(43) & 0.2(3) \\ \mathrm{C}(38)-\mathrm{C}(39)-\mathrm{C}(44)-\mathrm{C}(43) & -175.5(2) \\ \mathrm{C}(45) \# 1-\mathrm{C}(45)-\mathrm{C}(46)-\mathrm{C}(47) & -3.3(12) \\ \mathrm{C}(46) \# 1-\mathrm{C}(45)-\mathrm{C}(46)-\mathrm{C}(47) & -3.3(12) \\ \mathrm{C}(50)-\mathrm{C}(45)-\mathrm{C}(46)-\mathrm{C}(47) & -2(3) \\ \mathrm{C}(50) \# 1-\mathrm{C}(45)-\mathrm{C}(46)-\mathrm{C}(47) & 179(7) \\ \mathrm{C}(47) \# 1-\mathrm{C}(45)-\mathrm{C}(46)-\mathrm{C}(47) & -173(2) \\ \mathrm{C}(51)-\mathrm{C}(45)-\mathrm{C}(46)-\mathrm{C}(47) & 176.3(14) \\ \mathrm{C}(45)-\mathrm{C}(46)-\mathrm{C}(47)-\mathrm{C}(48) & -1(2) \\ \mathrm{C}(46)-\mathrm{C}(47)-\mathrm{C}(48)-\mathrm{C}(49) & 4.3(19) \\ \mathrm{C}(47)-\mathrm{C}(48)-\mathrm{C}(49)-\mathrm{C}(50) & -3.8(18) \\ \mathrm{C}(45) \# 1-\mathrm{C}(45)-\mathrm{C}(50)-\mathrm{C}(49) & 3.7(13) \\ \mathrm{C}(46) \# 1-\mathrm{C}(45)-\mathrm{C}(50)-\mathrm{C}(49) & 180(10) \\ \mathrm{C}(46)-\mathrm{C}(45)-\mathrm{C}(50)-\mathrm{C}(49) & 3(3) \\ \mathrm{C}(50) \# 1-\mathrm{C}(45)-\mathrm{C}(50)-\mathrm{C}(49) & 3.7(13) \\ \mathrm{C}(47) \# 1-\mathrm{C}(45)-\mathrm{C}(50)-\mathrm{C}(49) & -179.4(16) \\ \mathrm{C}(51)-\mathrm{C}(45)-\mathrm{C}(50)-\mathrm{C}(49) & -175.7(13) \\ \mathrm{C}(48)-\mathrm{C}(49)-\mathrm{C}(50)-\mathrm{C}(45) & 0(2) \\ & \end{array}$

Symmetry transformations used to generate equivalent atoms:

\#1 -x+1,-y,-z 


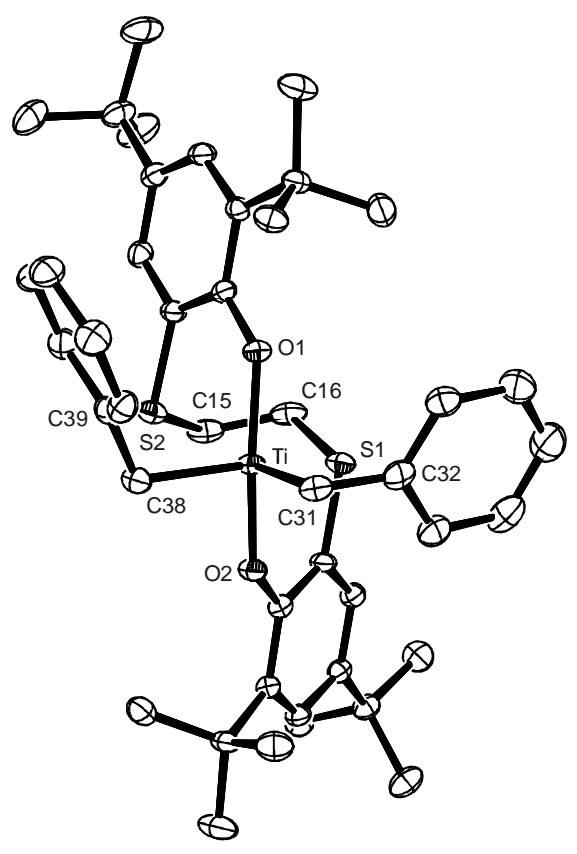

Figure S1. ORTEP diagram of the molecular structure of $\mathbf{2 b}$. Hydrogen atoms were omitted for clarity; thermal ellipsoids are drawn at the 50\% probability level. 


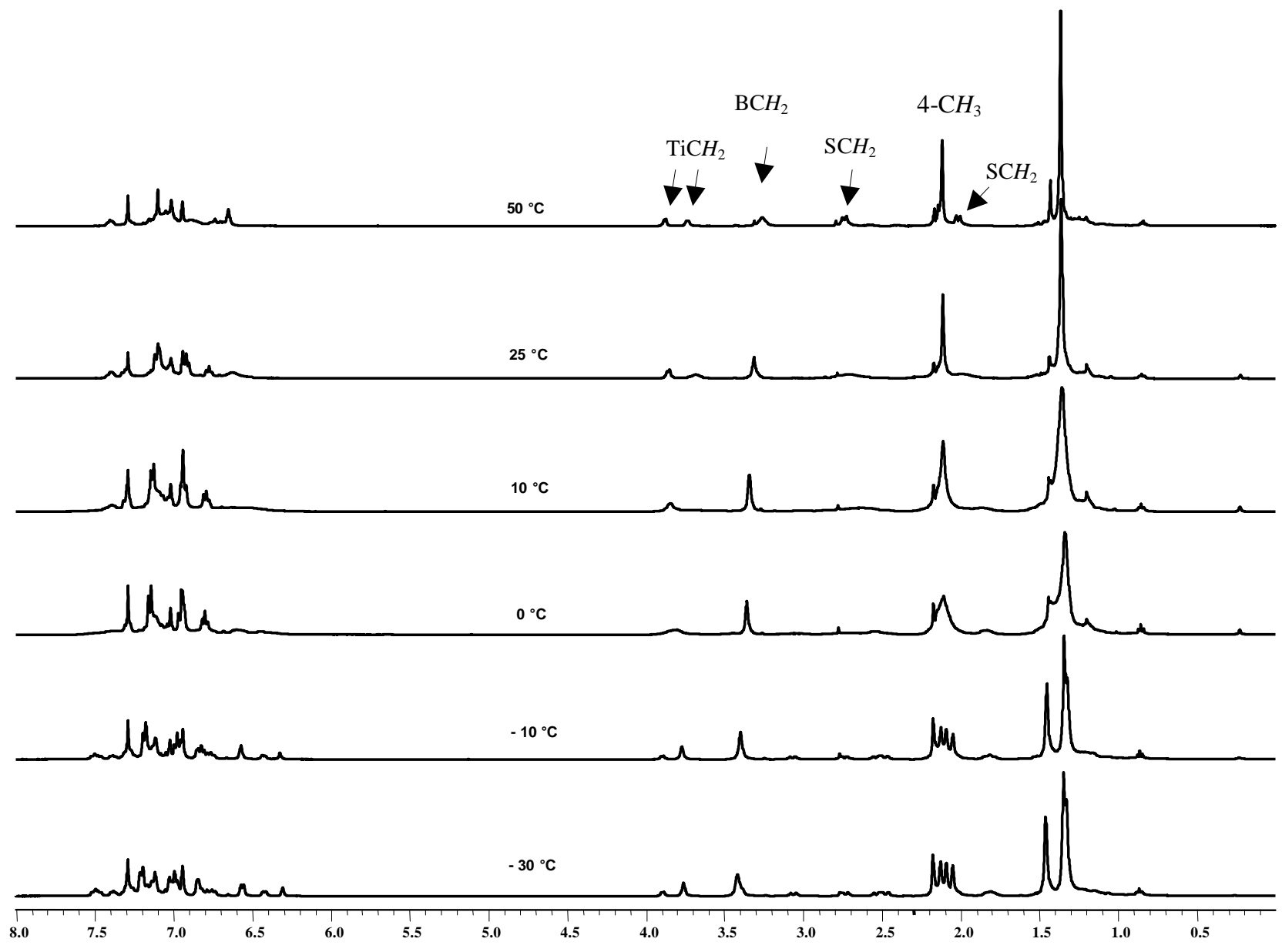

Figure S2a. Variable-temperature ${ }^{1} \mathrm{H}$ NMR spectrum of the reaction mixture of $\mathbf{2 a}$ with $\mathrm{B}\left(\mathrm{C}_{6} \mathrm{~F}_{5}\right)_{3}$. 


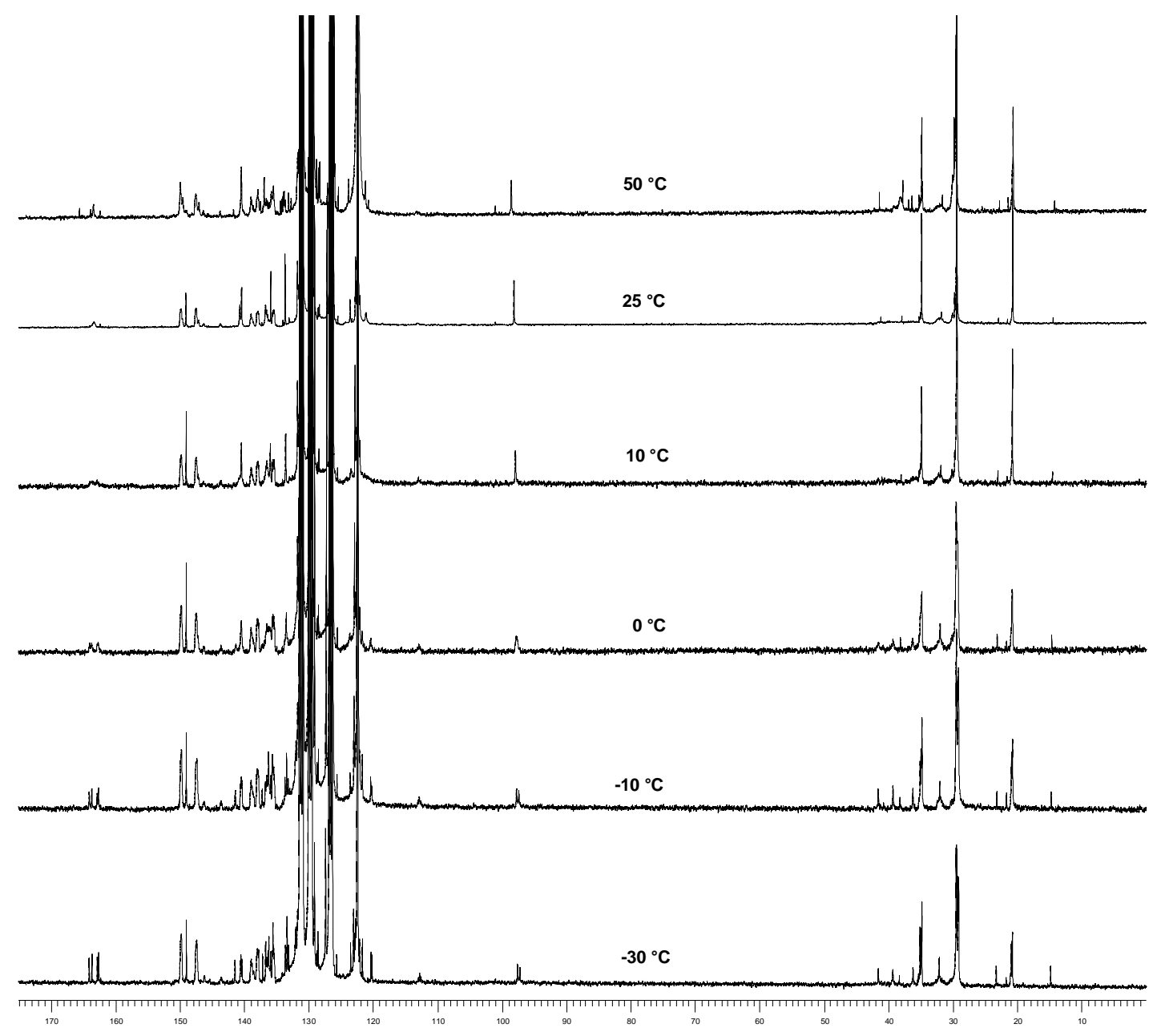

Figure S2b. Variable-temperature ${ }^{13} \mathrm{C}$ NMR spectrum of the reaction mixture of $\mathbf{2 a}$ with $\mathrm{B}\left(\mathrm{C}_{6} \mathrm{~F}_{5}\right)_{3}$. 
Figure S3a. ${ }^{1}$ H NMR spectrum of iPS (run 6, Table 3)

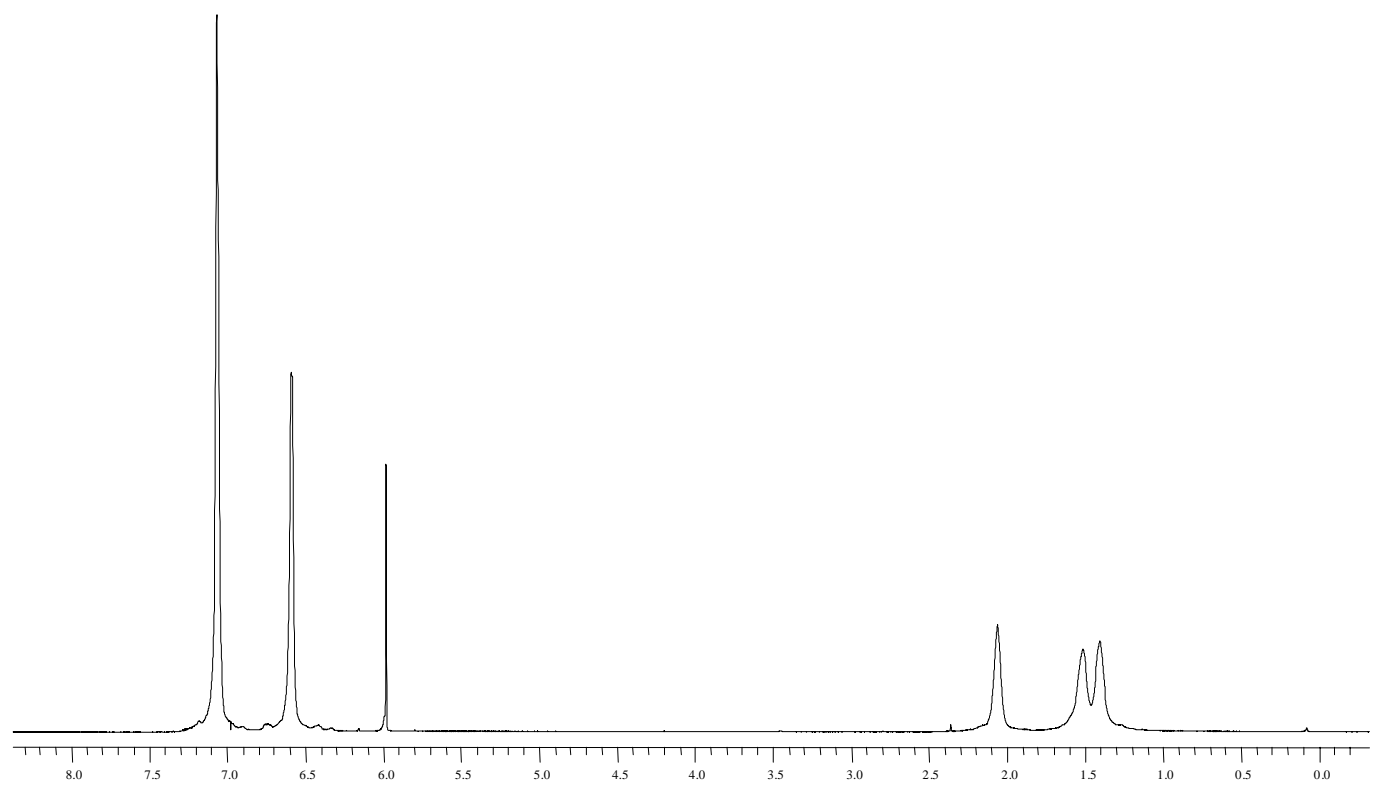

Figure S3b. ${ }^{13} \mathrm{C}$ NMR spectrum of iPS (run 6, Table 3)

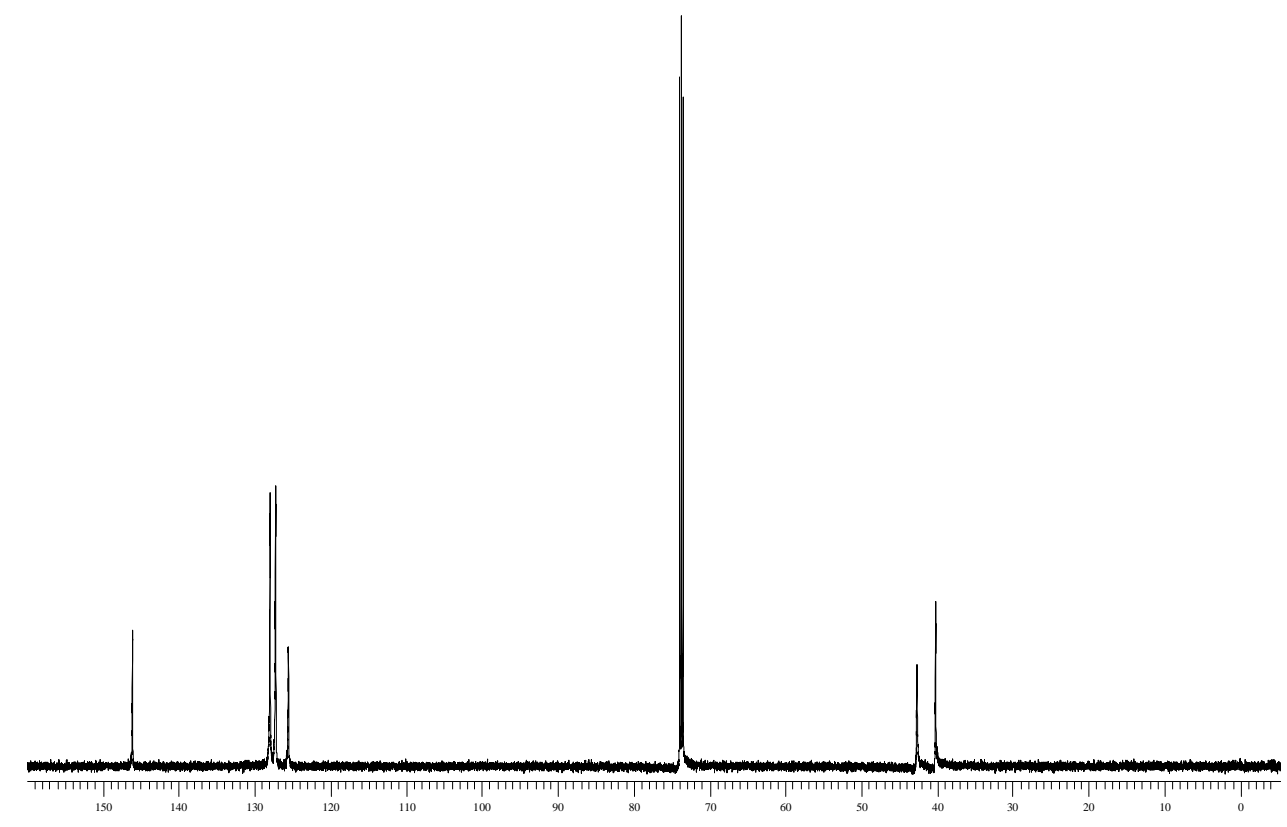

